\section{INVITED SPEAKERS ABSTRACTS}

\section{IS-01 \\ CHRONIC ACIDOSIS REWIRES CANCER CELL METABOLISM THROUGH PPARa SIGNALING}

Rolver, M.G. ${ }^{1}$, Holland, L.K.K. ${ }^{2}$, Prasad, N.S. ${ }^{1}$, Ponniah, M. ${ }^{1}$, Yao, J.3, ${ }^{4}$ Schnipper, J. ${ }^{5}$, Kramer, S. ${ }^{1}$, ElingaardLarsen, L. ${ }^{6}$, Pedraz-Cuesta, E. ', Liu, B. ${ }^{2}$; Pardo, L.A. ${ }^{7}$, Sandelin, A. ${ }^{3}{ }^{4},{ }^{*}$, Maeda, $\mathrm{K}^{2},{ }^{*}$, Pedersen, S.F. ${ }^{*},{ }^{*}$

${ }^{1}$ Department of Biology, University of Copenhagen, Denmark

${ }^{2}$ Cell Death and Metabolism, Center for Autophagy, Recycling and Disease, Danish Cancer Society Research Center, Copenhagen, Denmark

${ }^{3}$ The Bioinformatics Centre, Department of Biology, University of Copenhagen, Denmark

${ }^{4}$ Biotech Research and Innovation Centre, University of Copenhagen, Denmark

${ }^{5}$ University of Picardie Jules Verne, Amiens, France

${ }^{6}$ Steno Diabetes Center, Gentofte, Denmark

${ }^{7}$ Oncophysiology Group, Max-Planck-Institute for Experimental Medicine, Göttingen, Germany

Extracellular acidosis is one of the key hallmarks of solid tumors. Whereas another such hallmark, tumor hypoxia, has been widely studied, the mechanisms through which tumor acidosis impact cancer development and disease progression remain poorly understood. Using breast-, pancreatic- and colon cancer cells adapted to growth at extracellular $\mathrm{pH}$ (pHe) 6.5, we show that hundreds of genes are significantly up- or downregulated across three different cell types. Thus, a shared pattern of mRNA response to acidosis can be identified, albeit specific pathways differ between cell types. Acid adapted cancer cells increase expression of several net acid-extruding proteins, with the $\mathrm{Na}+, \mathrm{HCO} 3-$ cotransporter $\mathrm{NBCn} 1$ being upregulated in all cell types studied. Strikingly, cytosolic $\mathrm{pH}(\mathrm{pHi})$ remains acidic at pHe 6.5, yet becomes alkaline when cells are transferred to $\mathrm{pHe}$ values simulating the more favorable conditions outside the tumor core. Metabolically, the acid adaptationinduced phenotype is characterized by increased metabolic flexibility (Seahorse analysis), mitochondrial hyperfusion (superresolution microscopy), increased peroxisome- and lipid droplet content, elevated triacylglycerol content and increased fatty acid desaturation (shotgun lipidomics). Acid adaptation-upregulated genes and proteins are dominated by peroxisome proliferator-activated receptor- $\square$ (PPARA, PPAR $\square$ ) and its targets, including peroxisomal biogenesis factor-11 and carnitine palmitoyltransferases - supporting peroxisomal and mitochondrial lipid metabolism - and the metabolic regulator thioredoxin-interacting protein (TXNIP). PPAR $\square$ inhibition abolishes TXNIP upregulation, and TXNIP knockdown partially prevents acid adaptationinduced downstream events. We conclude that acid adapted cancer cells rewire metabolic pathways to rely more on fatty acid oxidation, yet that the adaptation process endows them with increased metabolic flexibility. This is at least in part dependent on acid induced upregulation of PPAR $\square$ - a key upstream regulator of metabolic changes - and favors cancer cell survival in acidic tumor niches.

\section{IS-02}

\section{ONCOCHANNELOPATHIES OF PROSTATE CANCER}

Charlotte Dubois, Fabien Vanden Abeele, V'yacheslav Lehen'kyi, Maylis Raphael, Pilar Flamenco, Loic Lemonnier, Gabriel Bidaux, Dimitra Gkika, Alessandra Fiorio Pla, Dmitro Gordienko, Roman Skryma and Natalia Prevarskaya $^{1}$

${ }^{1}$ Inserm U1003, Equipe labellisée par la Ligue Nationale Contre le Cancer, Laboratory of Excellence, Ion Channels Science and Therapeutics; Université Lille, France.

Ion channels constitute a novel area of research in oncology. Malignant transformation of cells is the result of enhanced proliferation, aberrant differentiation, and impaired ability to die resulting in abnormal tissue growth, which can eventually turn into uncontrolled expansion and invasion, characteristic of cancer. Such transformation is often accompanied by changes in ion channel expression and, consequently, by abnormal progression of the cellular responses with which they are involved. Moreover, tight association of cancer hallmarks with ion channels dysfunction gives a good reason to classify them as special type of channelopathies, namely oncochannelopathies. The relation of cancer hallmarks to ion channels dysfunction differs from classical definition of channelopathies, as disease states causally linked with inherited mutations of ion channel genes that alter channel's biophysical properties, in a broader context of the disease state. Regarding cancer hallmarks as oncochannelopathies departs from classical definition in one more essential aspect, namely, each cancer hallmark commonly depends from malfunction of not just one channel type, but from multiple channels, whereas classical channelopathies are traditionally viewed as one channel diseases.

At present, the expression profile of all calcium permeable channels and their isoforms in prostate carcinogenesis has not been yet completed while the specific functional roles for most of those are only just beginning to be understood. Here, we show that prostate cancer cells use calcium channel's protein redistribution as an oncogenic switch mechanism.

In particular, ORAI3 channels remodeling results from genomic and microenvironment perturbations that disrupt the equilibrium of channels and favors the formation of novel $\mathrm{Ca} 2+$ channels activated in a store-independent manner. This remodeling of $\mathrm{Ca} 2+$ signaling in turn induces cell progression to a more aggressive pro-proliferative phenotype. Our study specifically positions these channels at the center of molecular machinery linking dysregulated arachidonic acid metabolism, calcium homeostasis, and oncogenesis. 
TRPV6 is a highly selective Ca2+ channel which exercises its normal physiological function via $\mathrm{Ca} 2+$ absorption in intestine and kidney. Intriguingly, we show that TRPV6 channel may switch from its well-known constitutive activity to the store-operated due to the remodelling mechanism involving STIM1/Orail-induced activation of TRPV6 channel translocation to the plasma membrane via Ca2+/Annexin I/S100A11 pathway. Moreover, we demonstrate that the discovered mechanism is employed by prostate cancer cells. Void of this channel in health, prostate cancer cells express it de novo where it changes the role by supplying $\mathrm{Ca} 2+$ which is used in cancer to increase cell survival.

Voltage dependent Ca2+ channel, Cav3.2, is expressed exclusively in neuroendocrine differentiated prostate cancer cells where this channel regulates both basal and stimulated secretion of mitogens, the process which in turn, stimulates the proliferation of neighbouring epithelial prostate cancer cells.

We were also interested by TRPM8 channels since TRPM8 is a target gene of the androgen receptor and its expression strongly increases in prostate cancer. Recent evidence we have obtained indicate that TRPM8 may be expressed not just in the plasma membrane, but also in the endoplasmic reticulum (ER) membrane where TRPM8 may operate as an ER Ca2+ release channel. The "preferred" TRPM8 localization depends on epithelial cell phenotype (differentiated apical cells vs. non-differentiated basal cells) and on androgen status (androgen-dependent vs. hormone refractory). In this regard, our recent advances in the characterisation of the TRPM8 role in cell migration inhibition could reflect a defending character of the plasma membrane localized TRPM8 in prostate cancer invasion and angiogenesis thus explaining the high metastatic potential of androgen-independent cancer cells lacking TRPM8.

In this regard, the recent advances in the characterisation of the TRPM8 role in cell migration inhibition could reflect a defending, "Good" character of the full-length TRPM8 channel in prostate cancer invasion. On the other hand, we portrayed as "Bad" TRPV6, and Orai3, which show a pro-proliferative role in prostate cancer cells. Finally, in the "Ugly" stage of the neuroendocrine hormone refractory cancer, Cav3.2 enhances this the more aggressive phenotype of the prostate cells.

Our data allow to suggest Orai3, TRPM8, TRPV6 and Cav3.2 as regulators of in prostate carcinogenesis and speculate on their potential use in therapeutics. This study specifically positions calcium permeable channels at the center of molecular machinery linking deregulated tumor metabolism, calcium homeostasis, and oncogenesis.

\section{IS-03 \\ TARGETED SILENCING OF NAV1.5 CHANNEL SUPPRESSES CELL PROLIFERATION AND INVASION IN OVARIAN CANCER CELLS}

Mümin Alper Erdoğan

Izmir Katip Çelebi University, Faculty of Medicine, Department of Physiology, Izmir, TURKEY; UT MD Anderson Cancer Center, Department of Experimental Therapeutics, Houston, TX, USA

Ovarian cancer (OCa) is the leading cause of gynecological cancer related deaths. OCa is one of the most aggressive cancer and associated with poor prognosis and survival rates (5-year survival rates about 50\%). OCa cells have highly invasive metastatic phenotype due to mutations, altered signaling pathways and deregulated of control mechanisms and patients with metastatic disease have poorer prognosis (5-year survival is 20\%). The major reasons for patient death include significant intratumoral heterogeneity, early metastasis and development of resistance to currently used chemotherapeutics. Therefore, identification of novel molecular targets and therapeutics strategies are urgently needed to enhance the efficacy of current therapies and prolong patient survival. Ion channels are important signaling molecules expressed in a wide range of tissues where they have significant involvement in determining a variety of cellular functions: solute transport, volume control, enzyme activity, secretion, invasion, gene expression, excitation-contraction coupling and intercellular communication. Studies indicated that there are significant differences in the regulation and the function of ion channels between normal and cancer cells. Voltage gated sodium channels (VGSC) is a group of ion channels that has been correlated with OCa because of their higher expression in highly metastatic ovarian cancer cells. Importantly, VGSC activity contributes to many cellular behaviors integral to metastasis in breast and other cancers including OCA. In this study, we investigated the NaV1.5 as integral component of the metastatic process in human OCa. The aim of the current study was to reveal molecular mechanisms underlying the effects of NaV1.5 down-regulation and investigate the effects on $\mathrm{OCa}$ in vitro. In this study, the ovarian cancer cell lines (HEYA8, SKOV3-IP1, SKOV3-TR (taxol resistant)) were used. NaV1.5 and Control non-silencing small interfering RNAs (siRNA) were employed for therapy. As in vitro experiments, cell proliferation, colony formation, invasion, western blot analysis were performed. Our results showed that specific NaV1.5 siRNA treatments caused a significant reduction in cell proliferation, colony formation, and invasion capacity in OCa cells ( $p<0.0001$ ). To reveal molecular mechanisms underlying the effects of NaV1.5 down-regulation, we evaluated signaling pathways regulating cell proliferation and invasion/metastasis by western blot analysis and found that $\mathrm{NaV} 1.5$ expression promotes expression of PI3K/ AKT, Integrin $\beta 1 / F A K / S r c$ and P70S6K. In conclusion, our results suggest that Nav1.5 channels may contribute OCa tumorigenesis and metastasis through the upregulation of oncogenic pathways and serve as a potential therapeutic target in $\mathrm{OCa}$. 


\section{IS-04 \\ COVID-19 VACCINES AND CANCER}

Hakan Akbulut

Ankara University Cancer Research Institute, Ankara, Turkey

The COVID-19 pandemic affected the healthcare systems globally. One of the most vulnerable populations to the pandemic is cancer patients. In a recent global study that we have participated in and carried out at the beginning of the pandemic, it was shown that the pandemic has caused to stop cancer care by almost 50\% throughout the World (Jazieh AR et al., 2020). The incidence of severe events is almost 4-5 times more in cancer survivors and 5-7 times in patients with active cancer (Liang W et al., 2021). The patients on active cancer treatment are much more susceptible to severe events, including fatality. Due to the uncertainties regarding the diagnosis, course of the COVID-19, and the healthcare measurements the governments take to decrease the virus, this ratio was much higher in certain countries.

However, the safety measurements have loosened over time. However, the most relieving factor for the back to everyday life has become possible with COVID-19 vaccines. There are mainly four different vaccine platforms approved for emergency use in the COVID-19 pandemic: mRNA vaccines (BNT162b2 (PfizerBioNTech), mRNA-1273 (Moderna)), adenoviral vectored vaccines (AZD 1222 (Oxford-Astra Zeneca), Sputnik-V, AD26. COV2.S (Janssen), Convidecia (Cansino), inactivated vaccines (Coronavac (SinoVac), Hayat Vax (Sinopharm), Covaxin (Bharat BioTech), CoviVac (Chumakov Center)), and protein subunit vaccines (EpiVacCorona (VECTOR-Russia), RBD-Dimer (ZifiVax)) (Yusuf A et al., 2021). mRNA vaccines are mainly utilized in high-income countries (Yusuf A et al., 2021). On the other hand, the adenoviral vectored vaccines are the most widely used in the World. However, the rare thrombosis side effect of adenoviral vectored vaccines seen approximately every 300k-500k dose has caused some reservations for their use in cancer patients.

COVID-19 vaccines are effective in cancer patients

By the end of 2020, the COVID-19 vaccines have become available for routine vaccination programs. However, there were some concerns regarding the efficacy of vaccination in cancer patients, especially while on active treatment. However, previous reports have shown that cancer patients on active treatment might benefit from vaccination against viral diseases (Wumkes ML et al., 2013). In addition, cancer patients were among the vulnerable populations to be first vaccinated in society in high-income countries (Yusuf A, 2021).

The seroconversion rate in cancer patients may vary depending on the type of cancer, treatment, and vaccine. Almost all of the published studies regarding the COVID-19 vaccinations in cancer patients are based mainly on mRNA vaccines (Table). The seroconversion rates are usually between 30-95 \% (Table). Although seroconversion is not always translated into protecting SARS-Cov-2 infections, it is highly comparable to people without cancer. The cancer patients with solid who are not on active treatment have an almost similar strength of immune response against covid-19 vaccines. However, the strength of mRNA vaccines is low in patients with hematological malignancies (Monnin et al, 2021).

Likewise, the patients having cytotoxic treatment may also have lower immune response upon vaccination (Thakkar a et al., 2021). Surgery, radiotherapy, hormonal treatment, targeted therapies with tyrosine kinase inhibitors, and immune checkpoint inhibitors did not decrease the immune response during vaccination with mRNA covid-19 vaccines (Addao A et al., 2021 ). However, the patients on anti-CD20 MoAb treatment did not respond well to viral vaccines (Thakkar A., et al 2021).

Table: Studies on SARS-coV-2 spike antibody seroconversion rates after complete vaccination ( 2 dose) in cancer patients.

\begin{tabular}{|c|c|c|c|c|}
\hline Study & Cancer Type & $\begin{array}{l}\text { Num- } \\
\text { ber of } \\
\text { patients }\end{array}$ & $\begin{array}{l}\text { Vaccine } \\
\text { Type }\end{array}$ & $\begin{array}{l}\text { Serocon- } \\
\text { version } \\
(\%)\end{array}$ \\
\hline $\begin{array}{l}\text { Pallich } \\
\text { R et al., } \\
2021\end{array}$ & Solid tumor & 95 & BNT162b2 & 55 \\
\hline $\begin{array}{l}\text { Monin } \\
\text { L et al., } \\
2021 \\
\end{array}$ & $\begin{array}{l}\text { Solid tumor, } \\
\text { hematologic } \\
\text { malignancies }\end{array}$ & 24 & BNT162b2 & 87,5 \\
\hline $\begin{array}{l}\text { Barriere } \\
\text { J et al., } \\
2021\end{array}$ & Solid tumors & 42 & BNT162b2 & 95,2 \\
\hline $\begin{array}{l}\text { Thakkar } \\
\text { A et al., } \\
2021\end{array}$ & $\begin{array}{l}\text { Solid tumors, } \\
\text { hematologic } \\
\text { malignancies }\end{array}$ & 200 & $\begin{array}{l}\text { BNT162b2 } \\
\text { mRNA- } \\
1273 \\
\text { AD26. } \\
\text { COV2.S }\end{array}$ & $87-95$ \\
\hline $\begin{array}{l}\text { Ligum- } \\
\text { sky H et } \\
\text { al., } 2021\end{array}$ & Solid tumors & 326 & BNT162b2 & 88,9 \\
\hline $\begin{array}{l}\text { Massar- } \\
\text { weh A et } \\
\text { al., } 2021\end{array}$ & Solid tumors & 102 & BNT162b2 & 90 \\
\hline $\begin{array}{l}\text { Addao } \\
\text { A et al., } \\
2021\end{array}$ & $\begin{array}{l}\text { Solid tumors, } \\
\text { hematologic } \\
\text { malignancies }\end{array}$ & 51 & $\begin{array}{l}\text { BNT162b2 } \\
\text { mRNA- } \\
1273\end{array}$ & $93-95$ \\
\hline $\begin{array}{l}\text { Schroff } \\
\text { RT et al., } \\
2021\end{array}$ & Solid tumors & 53 & BNT162b2 & $\begin{array}{l}80 \text { (neu- } \\
\text { tralizing } \\
\mathrm{Ab} \text { ) }\end{array}$ \\
\hline
\end{tabular}

BNT162b2: Pfizer-BioNtech mRNA vaccine; mRNA-1273: Moderna mRNA vaccine, AD26.COV2.S: Janssen adenoviral vector vaccine

In conclusion, the Worldwide COVID-19 vaccination recommendations for cancer patients are as follows as of September 2021 (NCCN 2021):

-Patients with hematopoietic stem cell transplantation or CAR-T or CAR-NK cell therapies should be vaccinated three months post-transplantation. 
-Patients with hematologic malignancies receiving intensive cytotoxic chemotherapy should be vaccinated when absolute neutrophile recovery happens.

-Solid tumor patients or patients with hematologic malignancies not receiving intensive cytotoxic chemotherapy should be vaccinated whenever the vaccine is available.

\section{COVID-19 vaccines do not cause cancer}

One of the most argued issues regarding the covid-19 vaccination is the potential cancer-inducing effects of the covid-19 vaccines, especially mRNA vaccines. None of the approved vaccine platforms for COVID-19 has been shown to induce cancer progression in any trial. Most of the arguments are usually based on the misunderstanding of a study published in 2018. In their seminal paper, Lee SH et al. have shown that widespread intronic polyadenylation inactivates tumor suppressor genes in B- cell leukemia/ lymphoma (Lee SH et al. 2018). The excessive amount of mRNA's that might be responsible for the inactivation of tumor suppressor genes was the mRNA's coding truncated forms of tumor suppressor proteins though they do not have any structural mutations (Lee SH et al. 2021). There is no evidence showing the integration of mRNA to the host genome through vaccines in humans. The other platforms, including vectored vaccines, inactivated vaccines, protein subunit vaccines, have not been shown to induce any form of cancer in humans or animal studies.

\section{IS-05 \\ DEVELOPMENT AND EVALUATION OF VIRUS LIKE PARTICLE VACCINE AGAINST COVID-19 INFECTION}

\section{Ihsan Gursel $^{1}$, Mayda Gursel ${ }^{2}$}

${ }^{1}$ Bilkent University, Molecular Biology and Genetics Department, Ankara, Turkey

${ }^{2}$ Middle East Technical University, Department of Biological Sciences, Ankara, Turkey

Multiple highly effective COVID-19 vaccines have recently been approved for human use along with numerous candidates under clinical development. Continued emergence of new variants providing escape from existing neutralizing antibodies of the vaccinees raises concerns on anti-spikespecific protection. Targeting of other SARS-CoV-2 structural protein antigens, (i.e. membrane, nucleocapsid and envelope) in addition to spike could broaden S-dependent protection of vaccine potency.

Herein, the development and immunogenicity of SARS$\mathrm{CoV}-2$ virus like particle (VLP) vaccine that incorporates the 4 structural proteins of SARS-CoV-2 is presented. We describe the preclinical and phase I clinical development of a virus like particle (VLP) vaccine expressing the hexaproline prefusion stabilized spike in addition to nucleocapsid, membrane, and envelope ( $\mathrm{N}, \mathrm{M}$ and $\mathrm{E}$, respectively) proteins. techniques to construct vectors suitable for mammalian cell transfection that is capable of assembling and secreting VLPs composed of four structural SARS-CoV-2 proteins to the extracellular environment. These antigenic SARS-CoV2-like nanoparticles mimic the size and shape of the wild type virus, express the prefusion superstable conformation of the spike protein on the VLP surface and act as an immunoprotective COVID-19 vaccine.

The VLPs were then adsorbed to alhydrogel (alum) and adjuvanted with a K-type CpG ODN to boost both the humoral and cellular (Th1 cells and CTL) immunity. Using different animal models we show that the vaccine is immunogenic and immunoprotective against viral challenge, elicit a Th1 but not Th2 dominated response, and induce SARS-CoV-2 specific humoral immunity. These data suggest that VLPs expressing all four structural protein antigens of SARSCoV-2 are immunogenic and can protect animals from developing COVID-19 infection following vaccination.

\section{IS-06 \\ NATURAL KILLER CELLS IN CANCER IMMUNOTHERAPY}

\section{Tolga Sütlü}

Department of Molecular Biology and Genetics, Boğaziçi University, Istanbul, Turkey

Natural Killer (NK) cells are lymphocytes of the innate immune system that have an essential role in the immune response against tumors and virus-infected cells. The clinical potential of using NK cells for cancer immunotherapy faces many obstacles related to ex vivo expansion, genetic modification and antigen-specific targeting of NK cells. Our research focuses on solutions to these obstacles and aims to bring ex vivo expanded and/or genetically modified NK cells closer to clinical application.

We have previously optimized GMP-compatible systems that have the capacity to expand polyclonal and highly cytotoxic NK cells using automated bioreactors. Furthermore, we have investigated the innate immune responses triggered in NK cells upon lentiviral genetic modification and developed methods for efficient ex vivo gene delivery. Our efforts have resulted in an optimized stimulation and genetic modification process for NK cells that greatly enhances lentiviral gene transfer. We have also evaluated different approaches to retarget NK cell cytotoxicity by genetic modification either towards ligands of NK cell activating receptors identified on tumor cells or by chimeric antigen receptors (CARs) of $\mathrm{T}$ cell receptors (TCRs) targeted against tumor-associated antigens.

Taken together, our results demonstrate the feasibility of developing novel cancer immunotherapy protocols based on genetically modified NK cells. 


\section{IS-07}

IMMUNE REGULATORY MECHANISMS OF B CELLS IN NON-SMALL CELL LUNG CANCER

Deniz Duralı

İstanbul Medipol University, Research Institute for Health Sciences and Technologies (SABITA), İstanbul

Aim: The survival of patients with non-small-cell lung carcinoma (NSCLC) is dismal despite chemotherapy, radiotherapy and surgery. Therefore search of new and more effective therapies such as immunotherapy has been pursued. The efficient use of immunotherapy and discovery of new therapeutic molecules require the understanding of mechanisms of anti-tumoral immune response mechanisms. Recently it has been demonstrated that B cells can be divided into different functional sub-groups according to their cytokine profiles such as effector $(\mathrm{Be} 1, \mathrm{Be} 2)$ and regulatory $\mathrm{B}$ (Breg) cells and by these cytokines they affect the functions of $\mathrm{T}$ lymphocytes. In addition to the release of cytokines, B cells also affect the $\mathrm{T}$ cell response through the immune modulator surface molecules they express (such as CD28, PD-1, PD-L1).

With the progression of cancer, an immunosuppressive tumor microenvironment is formed that may confer immunosuppressive characteristics in B cells. Studies suggest that B cells, which have inhibitory effect on tumor immunity via their cytokines (such as IL-10, TGF $\beta$ and IL$35)$ or through the immune modulator surface molecules (such as PD-1 and PD-L1) they express. Tumor infiltrating B cells in NSCLC are yet to be explored according to their functional cytokine profiles and the immune modulatory molecules they express on their surfaces. For better understanding of the tumoral microenvironment-dependent effects on functional differentiation of B cell sub-groups and their biological effects, this project is not limited to the study in tumor tissue (TT), but also includes the non-tumor healthy lung tissue (HT), mediastinal lymph nodes (mLN) and peripheral blood (PB) and these four tissues will be studied in a synchronized manner for the first time in this project.

Material-Methods: Tissue samples were obtained by surgery from NSCLC patients. In B cells, extracellular molecules expressions were analyzed by the flow cytometer and cytokines by PCR methods. The molecular mechanisms of PD-L1 expression were also investigated after in vitro stimulation in B cells purified from peripheral mononuclear cells from healthy individuals. Coculture experiments were performed to examine the effect of $\mathrm{B}$ cells on $\mathrm{T}$ cell proliferation and its correlation with PD-L1 expression.

Results: The ratios of B cells in lymphocytes from NSCLC patient tissues and blood samples were investigated. B cells were found in $\mathrm{mLN}, \mathrm{TT}, \mathrm{PB}$ and HT (respectively $22.9 \pm 11.7 \%, 16.9 \pm 14.7 \%, 5.1 \pm 3.6 \%$ and $4.3 \pm 3.7 \%$ ). The percentages of B cells vary widely among patients. IL-10, IL-35-EBI3, IL-12p35 mRNA expressions were detected in
B cells of all tissues obtained from NSCLC patients.

In recently published studies PD-1+ and/or PD-L1+ regulator $\mathrm{B}$ cells have been described. In the light of these findings, PD-L1, PD-1 and CD28 expressions in B cells from TT, HT, mLN and PB samples were examined. Results showed that TT-B cells had significantly higher PD-L1 expression compared to HT-B, mLN-B and PB-B cells $(\mathrm{P}<0.033 ; \mathrm{P}<0.033 ; \mathrm{P}<0.044)$. No significant difference was found between TT-B, HT-B, mLN-B and PB-B cells in terms of PD-1 and CD28 expressions (for PD-1, respectively $\mathrm{P}<0.578 ; \quad \mathrm{P}<0.090 ; \mathrm{P}<0.268 ;$ for $\mathrm{CD} 28$, respectively $\mathrm{P}<0.290 ; \mathrm{P}<0.137 ; \mathrm{P}<0.073)$. Together, these experiments showed the presence of a subset of $B$ cells expressing PD-L1 on tumor tissue.

Recently described CD24 and CD38 as Breg markers were examined in PD-L1+ and PD-L1- B cells of TT. The results suggest that the $\mathrm{CD} 24+\mathrm{CD} 38+$ is predominantly found in the PD-L1+ B cell group compared to PD-L1- B cells (respectively $22.8 \%$ and $8.6 \%$ ).

We also investigated regulatory cytokine (IL-10, IL-35) mRNA expression in tumor infiltrating PD-L1+ and PD-L1$B$ cells. Our results showed no expression differences in IL10 and IL-35 between PD-L1+ and PD-L1- B cells.

The specific expression of PD-L1 in TT-B cells suggests that inducer factor(s) are present in this tissue, such as tumor antigens. Experimental studies to identify the factors triggering PD-L1 expression were performed on B cells purified from $\mathrm{PB}$ obtained from healthy individuals, due to the limited number of B cells in tumor samples. It was found that $\mathrm{BCR}+\mathrm{SCD} 40 \mathrm{~L}$ stimulation in PB-B cells increased PD$\mathrm{L} 1$ and $\mathrm{PD}-1$ expressions 25 and 8 times $(\mathrm{P}<0.005 ; \mathrm{P}<0.003)$. The MEK1/2 pathway is one of the most effective pathways in PD-L1 expression. On the other hand, it is known that the Mek1/2 factor is also active in the BCR signaling pathway. CD19+ B cells purified from PBMCs of healthy individuals were stimulated for 24 hours with the combination of (antiIgG+IgM)+sCD40L with MEK1/2 inhibitor U0126 1 hour before stimulation to test the MEK1/2 hypothesis. Treatment with MEK1/2 inhibitor decreased approximately 2 and 5 times PD-L1 and PD-1 expressions compared to those without inhibitor $(\mathrm{P}<0.001 ; \mathrm{P}<0.003)$.

B cells stimulated with $\mathrm{BHR}+\mathrm{SCD} 40 \mathrm{~L}$ inhibit $\mathrm{T}$ cell proliferation. This inhibition correlates with increased PDL1 expression.

Conclusion: A subset of B cells that express tumor tissuespecific PD-L1 were identified in our studies. The PD-L1 expression is triggered by the MEK1/2 pathway as a result of antigenic stimulation in B cells. PD-L1+ B cells can be affecting the $\mathrm{T}$ cell response.

Keywords: Tumor immunology, non-small cell lung cancer, immunoregulation, regulatory B cells, T cells, cytokine 


\section{IS-08}

\section{DEVELOPMENT OF TARGETED- THERAPIES FOR HIGHLY AGGRESSIVE SOLID CANCERS}

\author{
Bülent Özpolat \\ MD Anderson Cancer Center, Department of Experimental \\ Therapeutics, Division of Cancer Medicine
}

Cancer is one of the top two causes of deaths in the US and the world. Cancer therapy includes standard therapies such as surgery, chemotherapy and targeted therapies, by antibody and small molecule inhibitors, and immunotherapy (i.e., check point inhibitors, CAR-T cell therapy). More recently RNA-based targeted therapeutics were approved by FDA. Although there are about more than 100 targeted therapies, due to significant heterogeneity in patient tumors even in the same subtype of cancers, only faction of patient can benefit from targeted therapies due to lack of target expression in all patients. Therefore, gene targeting RNA therapies such as microRNA and siRNA may overcome difficulties seen by the use of small molecule and antibodies provide promising new avenue for targeting these oncogenes. After several decades of research finally some of the RNA targeted therapies emerged and are being tested in clinical trials. Over the last decade our studies have focused on two major oncogenomic kinases, such as KRAS and Elongation Factor-2 kinase (EF2K) that we identified as a major oncogenic driver and molecular target and validated it as a therapeutic target in various solid tumors including breast, pancreatic, lung, and ovarian cancers. The talk will also give background in targeted therapies used in cancer patients and development of novel targeted therapies using noncoding RNAs (siRNA and microRNA) and small molecule-based targeted therapies on the several highly aggressive cancers including triple negative breast cancer, pancreatic, and lung cancer.

\section{IS-09 \\ DEVELOPMENT OF TARGETED THERAPIES FOR EWING SARCOMA}

\section{Aykut Uren \\ Georgetown University Medical Center}

Ewing Sarcoma (ES) is an undifferentiated small round cell tumor that is mainly observed in children, adolescents and young adults. ES grows in and around bones and soft tissues. The cure rate for patients with localized disease is $60-70 \%$, although survivors face long-term complications related to current chemotherapeutic agents. If a patient presents with metastasis or recurrent disease, the survival rate drops to below $20 \%$. Therefore, there is a critical need for novel effective therapies. Two of the important and unique features of ES are the expression of the cell surface protein CD99 at high levels and the presence of tumor specific chromosomal translocation, which produces the EWS-FLI1 chimeric oncoprotein. Both proteins are critical for ES growth both in vitro and in vivo. We discovered first in class small molecules that can directly bind to CD99 and EWS-FLI1 proteins and inhibit their functions. EWS-FLI1 inhibitor is currently in Phase 2 clinical trial.
Keywords: Ewing sarcoma, CD99, EWS-FLI1

IS-10

TARGETING CANCER AT THE NUCLEAR PORE

Ramzi M. Mohammad

GI-Cancer Research Room 732, HWCRC

Wayne State University

Barbara Ann Karmanos Cancer Institute

Exportin 1 (XPO1), also known as chromosome region maintenance protein 1 , plays a crucial role in maintaining cellular homeostasis via the regulated export of a range of cargoes, including proteins and several classes of RNAs, from the nucleus to the cytoplasm. Dysregulation of this protein plays a pivotal role in the development of various solid and haematological malignancies. Furthermore, XPO1 is associated with resistance to several standard-of-care therapies, including chemotherapies and targeted therapies, making it an attractive target of novel cancer therapies. Tumor-suppressor proteins are inactivated by many different mechanisms, including nuclear exclusion by chromosome region maintenance (CRM)-1. Increased tumor levels of CRM-1 have been correlated with poor prognosis of patients with cancer, making it a therapeutic target. Selective inhibitors of nuclear export (SINEs) bind to CRM-1 to irreversibly inhibit its ability to export proteins; we investigated a new class of SINEs in pancreatic cancer cells. We studied the effects of SINE analogs in a panel of pancreatic cancer cell lines and nontransformed human pancreatic ductal epithelial cells using proliferation, apoptosis, immunoblot, coimmunoprecipitation, small inhibitor RNA, and fluorescence microscopy analyses. The effects of the SINEs also were investigated in mice with subcutaneous and orthotopic tumors. SINEs (KPT-185, KPT-127, KPT-205, and KPT-227) inhibited proliferation and promoted apoptosis of pancreatic cancer cells, but did not affect human pancreatic ductal epithelial cells. The nuclei of cells incubated with KPT-185 accumulated tumorsuppressor proteins (p27, FOXO, p73, and prostate apoptosis response-4 [PAR-4]) and inhibited interactions between CRM-1 and these proteins. Mutations in the region of CRM-1 that bind to SINEs (Cys-528), or small inhibitor RNA knockdown of PAR4, prevented the ability of KPT-185 to block proliferation and induce apoptosis of pancreatic cancer cells. Oral administration of KPT-330 to mice reduced growth of subcutaneous and orthotopic xenograft tumors without major toxicity. Analysis of tumor remnants showed that KPT-330 disrupted the interaction between CRM-1 and PAR-4, activated PAR-4 signaling, and reduced proliferation of tumor cells.

Conclusions: Over the years, a number of selective inhibitors of nuclear export have been developed. However, only selinexor has been clinically validated. Data from clinical trials have led to the approval of the XPO1 inhibitor selinexor. Nowadays, there are more than 70 clinical trials in various cancers using selinexor as therapeutic agent. 


\section{IS-11}

\section{DIETARY CONTROL OF STEM CELLS IN} PHYSIOLOGY AND DISEASE

\author{
Ömer H. Yılmaz \\ Koch Institute, Massachusetts Institute of Technology \\ (MIT)
}

Organismal diet has a profound impact on tissue homeostasis and health in mammals. Adult stem cells mediate many aspects of tissue adaptation by balancing self-renewal and differentiation divisions to alter tissue composition in response to the environment. Because somatic stem cells may respond to organismal physiology to orchestrate tissue remodeling and some cancers are understood to arise from transformed stem cells, these findings raise the possibility that organismal diet, stem cell function, and cancer initiation are interconnected. Here I will present work from my group that describes our emerging view of how diet, metabolites and nutrient-sensing pathways instruct intestinal stem cell fate in homeostasis, adaptation to diet and diseases such as cancer.

\section{IS-12}

\section{TARGETING PKLR FOR EFFECTIVE TREATMENT OF NAFLD}

\section{Adil Mardinoglu ${ }^{1}, 2$}

${ }^{1}$ Science for Life Laboratory, KTH - Royal Institute of Technology, Stockholm, Sweden

${ }^{2}$ Centre for Host-Microbiome Interactions, Faculty of Dentistry, Oral \& Craniofacial Sciences, King's College London, London, United Kingdom

To develop novel strategies for prevention and treatment as well as to gain detailed insights about the underlying molecular mechanisms of liver diseases, it is vital to study the biological functions of liver and its interactions with other tissues and gut microbiota. Biological networks can provide a scaffold for studying biological pathways operating in the liver in connection with disease development in a systematic manner. In my presentation, I will present our recent work where biological networks have been employed to identify the reprogramming in liver physiology in response to NASH/NAFLD. I will further discuss how this mechanistic modelling approach can contribute to the discovery of PKLR as a promising drug target which may lead to design of targeted and effective treatment strategies for NAFLD and associated diseases.

\section{IS-13}

\section{CONSIDERING CANCER AS A METABOLIC DISEASE:}

NEW HORIZONS AND ROADMAPS IN CANCER THERAPEUTICS

Mustafa Guzel ${ }^{1}{ }^{2}$

${ }^{1}$ Istanbul Medipol University, Research Institute for Health
Sciences and Technologies (SABITA), Center of Drug Discovery and Development, Beykoz/Istanbul, Turkey 34810

${ }^{2}$ Istanbul Medipol University, International School of Medicine, Department of Medical Pharmacology, Kavacik Campus, Kavacik-Beykoz/ISTANBUL 34810 mguzel@medipol.edu.tr

Cancer now surpasses heart disease as leading cause of death in many countries of the world. Clinical validations prove that protein kinases are an attractive class of therapeutic drug targets for cancer as demonstrated with the recent approval of six protein kinase inhibitors. The Warburg effect describes the particular reliance of cancer cells on glycolysis for energy. Increased glycolysis and acid resistance have been postulated to be an essential part of carcinogenesis, conferring a significant growth advantage as well as promoting typical tumor progression. Warburg hypothesized that the energy consumption of cancer cells is different than the normal cells. When compared to normal conditions, cancer cells do not undergo tricarboxylic acid (TCA) cycle therefore resulting in more lactate in the cells. Glycolysis pathway is a way of cancer cells to provide energy. Targeting accelerated glycolysis in cancer cells is a new promising modality for treatment of cancer. Inhibition of glycolysis can be done without significant side effects, and such treatment will be additive to most known cancer therapies. The first step in glycolysis is the phosphorylation of glucose to glucose-6-phosphate. This reaction is catalyzed by the hexokinase-II enzyme (HK-II) which is known to be overexpressed in tumor cells. The feeding of cancer cells can be prevented by inhibiting the hexokinase-II enzyme in the first step of aerobic glycolysis. In literature, Methyl Jasmonate (MJ) is known as a Hexokinase-II inhibitor since it disposes VDAC and HK-II interaction on mitochondrial membrane. In our study, we aimed to increase the activity by synthesizing the novel MJ analogues with appropriate modifications. Recent studies show that Methyl Jasmonate reveals promising results for treatment of cancer as a HK-2 inhibitor. Cis-jasmone, Jasmonic acid and Methyl jasmonate are cyclopentanones that are fatty acid derivatives. Jasmonates are plant stress hormones which exhibit abnormal anti-cancer activity [1]. Jasmonates induced suppression of cell proliferation and death in a variety of cancer cell lines and cytotoxicity to cervical cancer cells with almost no effect on normal primary human keratinocytes [2]. As a result of our research, although methyl jasmonate is long-known natural product, it has not well-studied as an anti cancer agent.

Based on the three different cancer cell lines we investigated, our novel MJ analogues proved to be more potent than the original molecule. Thus this research may provide more efficacious/novel HK-II inhibitors and may shed light to develop new anti-cancer agents. In our research laboratory, we designed and synthesized handfull of novel methyl jasmonate analogs 
$3^{\text {rd }}$ International Cancer and Ion Channels Congress 2021

[3]. We will highlight the biological activity of those novel analogs as anti-cancer agents during the oral presentation.

This project $(215 \mathrm{~S} 890)$ is funded by TUBITAK. We kindly appreciate for their support.

Keywords: Cancer Metabolism, Methyl Jasmonate, Novel drug design and development, Anti-cancer Agents
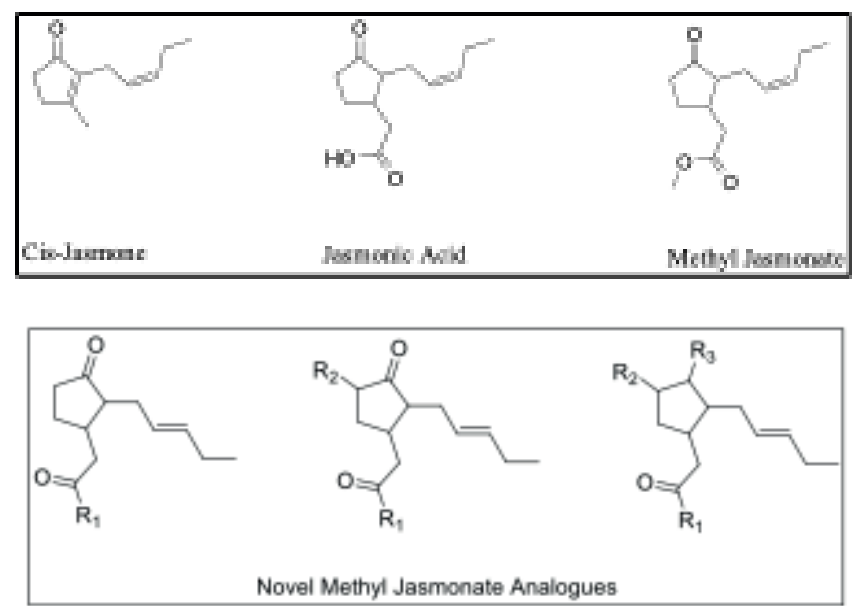

Figure 1: Cis-Jasmone, Jasmonic Acid, Methyl Jasmonate and Novel Methyl Jasmonate Analogs

\section{IS-14}

\section{AUTOPHAGY AND CANCER}

\section{Devrim Gozuacik}

Koç University Faculty of Medicine, Molecular Biology and Genetics Program and Koç University Research Center for Translational Medicine (KUTTAM), Istanbul, Turkey

Autophagy is key biological event that occurs at low basal levels in all cell types from yeast to mammals under non-deprived conditions, performing homeostatic functions such as protein degradation and organelle (e.g. mitochondria) turnover. It is rapidly upregulated during cellular stress, providing cells with recycled intracellular building blocks and substrates for energy generation and survival.

Autophagy dysregulation plays a critical role in the pathogenesis and progress of several human health problems, including neurodegenerative disorders (i.e. Alzheimer's, Parkinson's and Huntington's diseases), degenerative syndromes (i.e. Dystrophies and dystrophic syndromes), lysosomal storage disorders (i.e. Gaucher's disease), inflammation and cancer.

In our laboratory, we focus on the discovery of novel autophagy regulators and study implications of our findings in human disease pathogenesis and diagnosis. Moreover, we search for means to modulate autophagy for treatment purposes. In this speech, selected results of our research on autophagy and cancer will be discussed.
During the last two decades the remarkable progress has been achieved in cancer therapy, but primary or acquired drug resistance is the still main obstacle in successful treatments. Although different mechanisms considered that are responsible for tumor drug resistance, dysregulation of cellular energetic mechanisms are critical. AMP-activated protein kinase (AMPK) is a well-known major cellular energy sensor, which negatively regulates metabolic pathways. Cyclin-dependent kinase inhibitors (CDK) could lead to activation of AMPK, which led to differential responses in the cells according to their genetic differences. It was shown that CDK4 was a key coordinator between cellular metabolism and cell cycle progression. In our studies we determined that palbociclib and abemaciclib, CDK4/6 inhibitors, may lead to different responses on AMPK activation status. Especially lipid profile of the cells due to alterations on metabolic pathways were dysregulated according to AMPK activation status of pancreatic ductal adenocarcinoma (PDAC) cells following CDK4/6 inhibitor treatment. We have confirmed that AMPK silencing altered drug-mediated lipid biosynthesis pathways in MIA PaCa-2 cells. These alterations were not significant in PANC-1 PDAC cells. These preliminary findings showed that AMPK plays a critical role in CDK4/6 mediated cellular responses in pancreatic cancer cells through lipid biosynthesis pathways. Further research could enlighten the in vivo responses through monitoring lipid profile and fatty acid synthesis pathways, which are significantly altered in patients to evaluate therapy responses. Acknowledgement: This project supported by TUBITAK 1001 program (Project number 118z100).

\section{S-15}

\section{HSA-MIR-21-3P INFLUENCES THE PROGRESSION OF BREAST CANCER}

Arsalan Amirfallah ${ }^{16},{ }^{6 *}$, Hildur Knutsdottir ${ }^{2}$, Adalgeir Arason $^{3}, 6$, Bylgja Hilmarsdottir ${ }^{3}, 6$, Oskar T. Johannsson4, Bjarni A. Agnarsson ${ }^{5},{ }^{7}$, Rosa B. Barkardottir ${ }^{3},{ }^{6}$ and Inga Reynisdottir ${ }^{1}, 6$

${ }^{1}$ Cell Biology Unit, Department of Pathology, Landspitali - The National University Hospital of Iceland, 101 Reykjavik, Iceland

${ }^{2}$ Department of Biomedical Engineering, Johns Hopkins University, Baltimore, MD 21218, United States of America ${ }^{3}$ Molecular Pathology Unit, Department of Pathology, Landspitali - The National University Hospital of Iceland, 101 Reykjavik, Iceland

${ }^{4}$ Department of Oncology, Landspitali - The National University Hospital of Iceland, 101 Reykjavik, Iceland ${ }^{5}$ Department of Pathology, Landspitali - The National University Hospital of Iceland, 101 Reykjavik, Iceland ${ }^{6}$ Biomedical Center, Faculty of Medicine, University of Iceland, 101 Reykjavik, Iceland, 7Faculty of Medicine, University of Iceland, 101 Reykjavik, Iceland

Breast cancer is the most common cancer among women world-wide. It is a highly heterogeneous disease. 
Although the five-year survival rate is high, not all patients respond to therapy and some relapse after treatment. Due to heterogeneity of breast cancer and relapse of disease 6-10 years after diagnosis, it is crucial to find novel breast genes associated with progression of disease that may be used as diagnostic markers and as drug targets, like HER2. MicroRNA 21 is a known oncomir in various cancers, including breast cancer. It resides at $17 \mathrm{q} 23.1$ chromosomal region downstream of 3' UTR of VMP1 gene. MIR21 produces two mature products, miR-21-5p, which has been studied in detail, and miR-21-3p, which has received less attention. This study aimed to analyze if miR-21-3p expression was associated with characteristics that can predict patient prognosis. MiR-21-3p levels were quantified in two breast cancer cohorts, and its levels were correlated with clinical and pathological factors as well as the patients' survival. The results were followed up in two publicly available breast cancer cohorts TCGA and METABRIC. Correlation between miR-21-3p expression and mRNAs quantity in the patients' tumors were also analyzed and compared to miR-21-3p target genes from miRTarBase. The results indicate that high miR-21-3p expression is associated with worse prognosis for breast cancer patients and it affects pathways that support breast tumor proliferation, EMT and inflammation. However, additional work is needed to elucidate the association of miR-21-3p expression with distinct prognostic factors in breast cancer.

Keywords: MIR21, miR-21-3p, breast cancer, survival, prognosis, targets, pathways

\section{IS-16}

\section{INNOVATIVEAPPROACHESFORCONSTRUCTION} OF THERANOSTIC NANOPLATFORMS

\section{S. Sibel Erdem}

International School of Medicine, Medical Biochemistry, Istanbul Medipol University, 34810 Istanbul, Turkey, Research Institute for Health Science and Technologies (SABITA), 34810 Istanbul, Turkey

Despite the countless research and the revised treatment regimens, cancer is still a leading cause of death for millions of people. Diagnosis and treatment are more critical when the disease is at its early stage to reduce cancer related deaths. Since the existing systems for diagnosis of cancer are more appropriate for macro-sized tumors, micro tumors $(<1 \mathrm{~cm})$ can easily escape from detection leading metastases and/or recurrence of the disease. In addition, handling of the tumors that are resistant to various medications is another leading reason for ineffective treatment and undesirable outcome. Therefore, it is critical to construct new generation theranostic nano agents for early detection and treatment of the micro-sized tumors.

With the aim of detection and treatment of micro-sized tumors, inorganic nanoparticle-based nanoplatforms are being developed. These systems are specifically activated in the targeted tumor region and offer ultra-sensitive multimode imaging along with more than one type of treatment options. Multimode imaging such as Fluorescence Imaging, Magnetic Resonance Imaging and/or Computed Tomography ensures detection in more than one way to make tumor visible to the clinician. On the other hand, combination therapy targets to bring together local and systemic treatment protocols to act upon different cellular signaling and/or cell damage pathways to activate various mechanisms in the cell leading to death. Aforementioned new generation theranostic nanoplatforms will be opening a new avenue for real-time imaging and treatment of cancer. Research discussed here was supported by grants from the Scientific and Technological Research Council of Turkey, TÜBİTAK with grant numbers 119S219 and 118S299

Keywords: Fluorescence, micro tumors, Nanoparticles, Theranostic agent

\section{IS-17 \\ CAR-T CELL TREATMENT FOR PEDIATRIC PATIENTS WITH HAEMATOLOGIC MALIGNANCIES}

\section{S. Sema Anak}

İstanbul Medipol University, International School of Medicine Deptartment of Pediatric Hematology Oncology

In the last decade, treatment success of childhood leukemias, especially acute lymphoblastic leukemia, climbed over $80-90 \%$. But the treatment of the rest, relapsed/refractory leukemias, is still dismal. Together with other modalities including allogeneic hematopoietic stem cell transplantation (HSCT), chimeric antigen receptor (CAR)-T cell therapy has emerged as a promising treatment for this group in both children and adults. CD19directed CAR-T cell products have been licensed for use in paediatric B-cell acute lymphoblastic leukaemia that is refractory, in relapse post-transplant or in second or later relapses. Currently they are under investigation for frontline therapy for patients with poor-risk B-ALL: highrisk B-ALL with persistent minimal residual disease at the end of consolidation. Similar therapies for other lymphoid and myeloid malignancies are being developed for pediatric patients. Barriers, including antigen selection and toxicity, have prevented the rapid development of immune-based therapies for T-lineage and myeloid malignancies. For T-lineage diseases, targets have focused on CD5, CD7, and CD38, whereas myeloid disease targets have predominately focused on CD123, CD33, and, more recently, CLL-1. Other studies for pediatric solid tumors like brain tumors, neuroblastoma are underway with promising results. 


\section{IS-18 \\ CLINICAL ASPECTS OF CANCER RESEARCH}

Ömür Gökmen Sevindik

İstanbul Medipol University, Medipol Mega University Hospital, Hematology Department, Turkey

Today, there is an important paradigm shift in cancer treatment. Especially the transition from the conventional (traditional) chemotherapy era to the era of targeted therapy is perhaps the most important element of this paradigm shift. Based on the peak rate in 1991, it is possible to say that thanks to bio-pharmaceutical developments, a clear and sharp decrease of more than $30 \%$ was observed in cancer death rates. More than 1,300 drugs are currently in development, and the FDA has approved 115 cancer treatments in the last 20 years. The most important benefit of the age of targeted therapy has been that we can now offer our patients the luxury of being able to pronounce a permanent cure in a chronic disease such as cancer, with an incomparable "less side effect profile". Of course, this important movement and treatment evolution; It is an undeniable fact that we owe translational research, that is, translational medicine and research practice, which is well-designed and brings together pre-clinical and clinical science schools. If we want to increase our success in cancer treatment, we must master the biology of cancer, analyze the pathophysiological pathways, genomic foundations that have undergone serious breakthroughs in recent years, and reveal the potential gains that we can achieve by integrating these analyzes into existing treatment modalities or by using them as data in the development of much more innovative treatment models. "Cancer Clinical Research" lies at the heart of all these processes. Clinical research can be described as the presentation of the cooked food to the kitchen, especially in preclinical processes. This presentation process consists of Phase 1, which aims to determine the toxicity profile and ideal dose and administration scheme of the "innovative approach" in human cancers, Phase 2, which now aims to determine the toxicity concern, and Phase 2, where the net efficacy is examined, mostly single-arm, and the "innovative approach, which has proven its reach in singlearm studies." " can be summarized as Phase 3 studies that included much larger patient groups compared to the traditional approach. In fact, we are all familiar with this general concept of Phase and its definitions to a certain extent. However, when you get into these processes, the description of which is perceived as quite ordinary and simple, you see that it is actually so difficult to optimize the process. In particular, Phase 1 cancer research can be suggested as the stage in which pre-clinical medicine and clinical medicine schools experience the most interaction. Especially at this stage, where clear pharmacodynamic and pharmacokinetic analyzes are deeply involved, the primary principle of medicine, the "first do no harm" approach, comes to life and the potential dose-limiting features in almost every patient are tried to be documented in great detail. Similar concerns may be reflected in further phase studies with gradually decreasing intensity. Why are clinical trials, especially early phase clinical trials, valuable and more than valuable in the field of cancer? We can summarize the most important contribution as the possibility of offering our patients a new treatment option quite early in a situation where there is an "unmet clinical need", perhaps at the point where we feel most helpless, that can offer the opportunity to meet that clinical need in cell studies or cancer models.

Many pharmaco-economic parameters can seriously limit the chance of evaluating each new, effective treatment option with much less risk of toxicity, both in our country and in other countries. There are two ways to circumvent this constraint. Either to act as a manufacturer and developer with high $R \& D$ investments, or to meet with industry stakeholders in the role of manufacturer and developer on the axis of clinical studies in order to offer maximum benefit to your patients. When we examine the data of 2020, there are 16720 industry-supported active clinical studies $(23.1 \%$ Phase 1, 42\% Phase 2, 24.5\% Phase $3,10.4 \%$ Phase 4 ), while this number is unfortunately 521 in our country, $77.5 \%$ of which is Phase 3 studies. limited to working. There are many potential explanations for our country's slow momentum in cancer research. At the beginning of these, it can be counted that clinicians and pre-clinicians, both pre-clinicians and clinicians have negligible ties with government or private sector industry stakeholders, clinical research culture has not yet become an established culture. Behavioral reservations of our patients or their relatives can still be considered as the primary factor within the limitations of these studies, which are designed by considering very high ethical values, especially in the foot of the patient. In our country, the most important tasks that will pave the way for development in cancer clinical studies in order to observe the maximum patient benefit; We can summarize that it is to develop the laboratory culture of clinicians, to meet the clinical notion of pre-clinicians, to raise awareness of the severe consequences of unmet clinical need in the field of cancer, to focus the attention of industry and industry in this area and to ensure harmonization.

1. https://www.phrma.org/resource-center/Topics/Cancer/ The-Value-of-Cancer-Treatment-Today

2. PhRMA Annual Membership Survey 2020

\section{IS-19 INHIBITORS IN MALIGNANCIES}

IMMUNOTHERAPY: IMMUNE CHECKPOINT

Hüseyin Saffet Beköz

İstanbul Medipol University, Medipol Mega University Hospital, Hematology Department, Turkey

Immunotherapy has changed the way of cancer treatment for many patients. Unlike most other cancer treatment tools that directly target the tumor cells, 
the goal of immunotherapy is activate the patient's immune system to attack the cancer cells, just as it would a foreign invader.

Agents that affect the immune system have been used in cancer for decades. Immunotherapy truly broke new ground in 2011 with the approval of the first immune checkpoint inhibitor (ICI) ipilimumab, a monoclonal antibody that binds to cytotoxic $\mathrm{T}$ lymphocyte - associated protein 4 (CTLA-4) on T cells.

CTLA-4 acts as a brake on T cell activity. Briefly, when $\mathrm{T}$ cells encounter an antigen presenting cell (APC), two signals are required to stimulate $\mathrm{T}$ cell proliferation: binding of $\mathrm{T}$ cell receptor with major histocompatibility (MHC) complex found on APCs and binding of CD28 on T cell to B7 on APC.

Simultaneous activation of these signals induces $\mathrm{T}$ cell proliferation but also turns on CTLA-4 expression. As CTLA-4 accumulates on the surface of T cell, it outpaces CD28 for B7 and stops $\mathrm{T}$ cell proliferation. Targeting CTLA-4, ipilimumab shuts down one of the inhibitory pathways that blocks effective antitumor responses.

The other class of ICI targets programmed cell death protein 1 (PD-1) and its ligand programmed death ligand 1 (PD-L1), which form a distinct inhibitory pathway. Also, PD-1 expressed on T cells inhibits T cell activation upon binding to PD-L1 on the surface of APCs. In addition, some tumor cells express PD-L1 as a strategy to escape immune surveillance. Blocking PD-1 or PD-L1 can therefore increase $\mathrm{T}$ cell response.

The first anti-PD-1 treatments, pembrolizumab and nivolumab, entered the clinic in 2014 with U.S. Food and Drug Administration (FDA) approval. Since then, several other anti-PD-1/L1 treatments have been approved.

ICIs are now approved for a variety of solid tumors as well as several hematological malignancies. Given that antiCTLA-4 and anti-PD-1/L1 agents exert their antitumor effects through different mechanisms, they have also been used in combination. The introduction of ICIs into clinical practice has significantly reduced cancer-related mortality. A recent study showed that the overall mortality rate for metastatic melanoma decreased by $18 \%$ from 2013 to 2016, undoubtedly due in part to approval of ICIs.

However, while these agents have the potential to achieve lasting responses and perhaps even cures, many patients do not respond to treatment. In a long-term analysis of ipilimumab in metastatic melanoma, approximately $20 \%$ of patients achieved 3-year survival.

Although response rates can be improved with combination therapy, additional toxicity remains a concern.

In addition, despite the ever-growing list of approved indications for ICIs, many patients have cancers that either do not express immune checkpoint proteins or do not respond to ICIs. A retrospective, cross-sectional analysis of US cancer patients estimated that approximately $37 \%$ were candidates for ICI therapy in 2019.

It is clear that to increase the number of patients who can benefit from ICI therapy, it is necessary to identify the factors and biomarkers involved in the ICI response, develop strategies to increase response rates, and address tumor types that are resistant to ICIs.

\section{IS-20 \\ CIRCADIAN CLOCK AND CANCER}

Halil Kavakli

Koç University, Chemical and Biological Engineering, Molecular Biology and Genetics Rumeli Feneri Yolu Sarıyer İstanbul

The circadian rhythm is a biological signaling mechanism that oscillates with an internal period of exactly 24 hours with the signal they receive from the environment. Gene expression, cell division, and DNA repair are regulated by the clock, leading to the hypothesis that individuals with circadian clock disorders may predispose to cancer. At the molecular level, the circadian rhythm is generated by the negative and positive loops as a result of the interaction between of clock proteins. In summary, CLOCK, one of the main clock proteins, forms a dimer by interacting with the BMAL1 protein and initiates the expression of clock-controlled genes including Cryptochromes (Cry) and Period (Per) genes, by binding to the 6 nucleotidelong E-box located in the promoter region. CRY and PER proteins, which accumulate in the cytoplasm over time, form a triple complex with CKIepsilon and translocate into the nucleus, inhibit BMAL1/CLOCK-transactivation. In recent years, studies with mice have shown that in the absence of CRYs, cancer cells are directed to death by activating the apoptosis pathway in p53-induced cancer cells. In this talk, I will discuss our recent data on discovery of small molecules specifically regulates the activity of the CRY1 using structure-based drug design methods. Then I will talk about full characterization of a molecule, named as M47 by presenting in vitro, pharmacokinetic and pharmacodynamic experimental results. Finally, the effect of the M47 on life span of p53 null mutant mice will be further discussed.

\section{IS-21 \\ BIOLOGICAL CLOCK AND DNA DAMAGE RESPONSE}

Nuri Öztürk

Gebze Technical University, Molecular Biology and Genetics, Gebze, Kocaeli

Circadian rhythms are endogenous and autonomous 24hour oscillations created by the biological (circadian) clock. 
At the molecular level, the biological clock is created as follows: The transcription factors BMAL1 and CLOCK activate circadian clock-controlled genes (CCGs), and then products of two of these CCGs, CRYPTOCHROME and PERIOD, inhibit BMAL1/CLOCK activity. This activation and inhibition generates an oscillation that is set to about 24 hours by other pathways and post-transcriptional regulation mechanisms to regulate many daily events through activities of the products of CCGs. Disruptions in the biological clock are associated with cancer, neurological diseases and metabolic disorders. On the other hand, DNA damage response is essential for the continuation of normal cell viability, and its defects are associated with the conditions such as cancer and premature aging. Recently, it has been demonstrated that the DNA damage response is controlled by the biological clock. Although statistical data show that the disruption of the biological clock increases cancer incidence, unexpected results were obtained with mouse models. In order to better interpret these unexpected results, new DNA damage response factors that interact with the biological clock need to be discovered. In this talk, after discussing the molecular interactions between the biological clock and the DNA damage response, new approaches will be discussed on how to discover unknown interactions and to discover new proteins that can be targets for therapy. These new approaches are expected to guide the development of new mechanism-based chemotherapeutic regimens.

\section{IS-22 \\ NANOSCALE FLUOROPYRIMIDINE POLYMERS FOR OVERCOMING LIMITATIONS OF EXISTING THERAPIES FOR GASTROINTESTINAL MALIGNANCIES}

\author{
William H. Gmeiner \\ Wake Forest University School of Medicine \\ Winston-Salm, NC 27157 USA
}

Fluoropyrimidine drugs (FPs; e.g. 5-Fluorouracil (5-FU)) are used to treat $>2$ million cancer patients including most patients with gastrointestinal malignancies. While 5-FUbased regimens improve survival, high mortality rates are still associated with advanced cancers and many patients treated with FPs experience serious systemic toxicities that may be life threatening. Nanotechnology approaches have potential to improve the therapeutic index of FPs by altering the pharmacokinetic and pharmacodynamic properties of FPs.

My research program focuses on the development of nanoscale FP polymers (e.g. CF10) to reduce systemic toxicities and improve efficacy of FP chemotherapy. A major limitation of 5-FU is its inefficient conversion to the primary active metabolite, 5-fluoro-2'-deoxyuridine5'-O-monophosphate (FdUMP), which inhibits TS and is central to the anti-tumor and anti-metastatic activities of FPs. $>85 \%$ of 5 -FU is either degraded or excreted intact, while $<5 \%$ is metabolized to FdUMP. CF10 is a polymer of FdUMP, modified with the anti-cancer drug Cytarabine at the 3'-terminus and with short-chain polyethylene glycol (PEG5) to decrease polymer degradation by nucleases. CF10 can overcome many limitations of 5-FU because it is more directly converted to FdUMP, resulting in strong TS inhibition and also Topoisomerase 1 cleavage complex (Top1cc) formation. CF10 also causes lower systemic toxicities than 5-FU because toxicities result from ribonucleotide metabolites that are produced at much lower levels with CF10.

Recent studies have demonstrated significant efficacy advantages for CF10 relative to 5-FU in mouse models of gastrointestinal malignancies. In an orthotopic CRC tumor model using HCT-116-luc cells in Balb/c nu/nu mice treated with CF10 (2x/wk; $300 \mathrm{mg} / \mathrm{kg})$ displayed stable weights and on average gained weight with treatment extending to 8 weeks. Mice treated with CF10 also remained active throughout the study, with no signs of drug-related toxicity. Analysis of tumor growth rates showed the CF10 group had significantly reduced tumor growth than either 5-FU or vehicle $(p=0.014)$. When we examined survival times among groups, we found a highly significant difference based on the Log-Rank test (chi-square 19.8, 2 degrees of freedom $\mathrm{p}<0.0001$ ) - with the CF10 animals having much longer survival (median 84.5 days) than control or 5-FU (33 days and 32 days, respectively). Our results demonstrate CF10 increased survival in the HCT-116-luc orthotopic model, while 5-FU had no significant effect. We have also demonstrated anti-tumor activity for CF10 in flank tumor models of colon cancer and pancreatic cancer and demonstrated promising anti-metastatic activity. Collectively our studies demonstrate CF10 has potential to overcome limitations of 5-FU in treating GI malignancies and justify initiation of a clinical trial.

\section{IS-23}

\section{THE ROLE OF IMMUNOSUPPRESSIVE MYELOID CELLS IN TUMOR MICROENVIRONMENT AND PRE-METASTATIC NICHE}

Fulya Alkan Esra Altintas, Ayca Onal, Raziye Piranlioglu, EunMi Lee, Hasan Korkaya

Georgia Cancer Center, Biochemistry and Molecular Biology, Augusta University

Although metastasis is an extremely inefficient process that entail overcoming multiple barriers including anti-tumor immunity. Despite evidences from preclinical and clinical settings suggest that dissemination of malignant cells is an early process, majority of disseminated cells either eliminated or remain dormant in distant organs, while very few cells eventually develop successful metastasis. Therefore, it is widely accepted that dynamic and reversible tumor cell plasticity is required for metastasis, however, in vivo steps and molecular mechanisms are poorly elucidated. 
It is well known that clinically apparent metastasis is associated with late stages of cancer development, whereas micro-metastatic dissemination may be an early event. However, the fate of these early disseminated tumor cells (DTC) remains elusive.

Using the syngeneic mouse models, we demonstrated that although both orthotopically-implanted murine 4T1 and EMT6 tumors are capable of disseminating into secondary organs, only 4T1 tumors develop overt metastasis. However, EMT6 tumors induce an anti-tumor immunity in syngeneic mice and that eradicates disseminated tumor cells (DTC) in distant organs. Following the complete removal of primary EMT6 tumors, mice do not develop detectable metastasis and generate an immunological memory that leads to complete elimination of repeatedly injected tumor cells via tail vein. Conversely, these cells readily grow and metastasize in immuno-deficient athymic or Rag2- mice, and when g-MDSCs from 4T1 tumor-bearing mice were co-injected into immunocompetent EMT6 primed mice. In contrast to complete resection, mice with residual tumors following surgery exhibited an enhanced growth of local and concomitant growth of DTCs at metastatic site with increased g-MDSCs accumulation in lung and spleen.

Together, our results suggest that some tumors are capable of inducing anti-tumor immunity against the DTCs when complete resection of primary tumor cures animals. However, in the presence of residual tumors, inflammation induced by surgical procedure promote the growth of DTCs.

\section{IS-24}

\section{ADIPOSE NICHE REPROGRAMS THE METASTATIC MACHINERY OF CANCER CELLS}

\section{Giorgio Stassi}

University of Palermo, Department of Surgical and Oncological Sciences

Obesity is a strong risk factor for cancer progression, posing obesity-related cancer as one of the leading causes of death. Nevertheless, in obese patients, the molecular mechanisms that endow cancer cells with metastatic properties are not conclusively comprehended. Here, we show that visceral adipose tissue provokes a transition in the transcriptomic expression profile of cancer stem cells derived from CMS2 (epithelial/canonical consensus molecular subtype) colon cancer patients towards a mesenchymal subtype (CMS4). Adipokines IL-6 and HGF, secreted by visceral adipose tissue, activate STAT3, which inhibits miR-200a expression and as a result enhances ZEB2, effectively reprogramming CMS2 colon cancer stem cells into a mesenchymal-like phenotype. Notably, STAT3 pathway inhibition reduces ZEB2 expression and abrogates the metastatic growth sustained by adipokines. Importantly, in a colon cancer patient cohort, high expression levels of ZEB2 predict decreased survival probability. Together, our data suggest that targeting adipokines in obese CMS2 colon cancer patients may represent a therapeutic strategy for preventing metastatic disease.

\section{IS-25}

\section{DEVELOPMENT OF THERAPEUTIC SYSTEMS TARGETING TUMOR MICROENVIRONMENT IN LUNG CANCER}

Abdullah Karadag, Sinem Nalbantoglu, Dilek Ceker, Altan Kara, Kemal Sanli, Sevde Kucuker, Tugba Okyay, Saime Surmen, Mustafa Surmen, Aydan Sarac, Tugba Kul Koprulu, Omer Kacar, Ali Aydin, Arslan Ali, Melike Dinc, Mustafa Beker, Yusuf Tambag, Mucahit Buyukyilmaz, Ali Osman Cibikdiken, Serdar Evman, Volkan Baysungur, Abidin Sehitogullari, Ahmet Midi, Sadrettin Pence, Mahmut Gumus, Ihsan Boyaci, Neslin Emekli, Saban Tekin, Kazım Yalçın Arga

TÜBITTAK, Turkey

Management of lung cancer is challenging as the disease is characterized with poor prognosis due to high incidence and death rates because of diagnosis at advanced stages when the majority of patients are associated with local or distant metastasis. Local resection of the tumor is the most effective treatment, but majority of the patients are not eligible. Chemoradiotherapy, immunotherapy, and targeted therapy are alternative options; however, rapid development of resistance usually results in relapse and poor prognosis. There is a huge need for the development of new screening and/or diagnostic systems to diagnose patients at the early stages as well as novel monitoring systems to identify the development of drug resistance and recurrence immediately. Furthermore, despite recent progresses in targeted therapy and immunotherapy, development of new therapeutic systems for the management of this deadly disease is critical. We have developed a research program to challenge this deadly disease via identification of an associated molecular profile at various levels (DNA, RNA, protein, metabolite, etc.) through an advanced bioinformatics-supported integrative multiomics approach. Specimens from normal lung tissue as well as primary tumor, tumor microenvironment, and lymph node metastasis alongside body fluids have been obtained through an approved IRB and patient consent. Tissue samples were snap-frozen immediately, then transferred to the local biorepository system through cold-chain and kept at $-80 \square$ until being further processed. Tissue samples were homogenized to powder form and then aliquot for either DNA or RNA isolation and characterization for whole genome or total transcriptome analysis through nextgeneration sequencing, respectively, or further processed for proteome or metabolome analysis through LC-MS/MS. Likewise, blood/serum/plasma samples were processed for the multiomics analysis. Data were analyzed through advanced bioinformatics to identify molecular profile associated (at various levels) to the disease. 
Each profile needs to be verified on large patient cohorts and appropriate control samples. Furthermore, target molecules have been / are being identified through bionetwork analysis for the development of therapeutics as well as vaccines in the future. Early analyses indicate that targeting tumor microenvironment could be as critical as tumor tissue itself. Our novel findings through integrative multiomics approach may pave the way for the development of efficient screening, diagnosis, monitoring, and therapeutic systems as well as improved management of lung cancer.

\section{IS-26 \\ ENDOGENOUS BIOELECTRIC NETWORKS: FROM BASIC MECHANISMS OF CANCER SUPPRESSION TO ELECTROCEUTICALS}

\author{
Michael Levin ${ }^{1}, 2$, \\ ${ }^{1}$ Allen Discovery Center at Tufts University \\ ${ }^{2}$ Wyss Institute, Harvard University
}

Why are there ever any organs that are not tumors? Normal tissue and organ structure requires individually competent cells to cooperate towards large-scale anatomical goals. One important system by which cells achieve anatomical homeostasis is bioelectric signaling. All cells, not just neurons and muscle, use ion channels and electrical synapses known as gap junctions to form networks that process information. These bioelectric networks set up spatio-temporal patterns of resting potentials that guide morphogenesis during embryonic development, regeneration, and cancer suppression in adulthood. Stressors, including oncogenes, can cause cells to disconnect from these electrical networks, driving them toward a primitive unicellular state in which they treat the rest of the body as external environment (resulting in metastasis and attempts to regain multicellularity as tumors). In this talk, I will introduce developmental bioelectricity, and how how manipulation of bioelectrically-mediated information can be used to induce cancer (in the absence of genetic damage), detect cancer (by non-invasive voltage imaging), and suppress or normalize cancer despite potent oncogenes. New machine learning strategies are coming on-line to identify drugs that manipulate bioelectric states aay from cancer and towards normal organogenesis and tissue maintenance.

Keywords: bioelectricity, Vmem, voltage, resting potential, electroceutical, ion channel

\section{IS-27}

\section{ION CHANNEL COMPLEXES: A NEW PARADIGM IN CANCER RESEARCH}

\section{Annarosa Arcangeli}

Department of Experimental and Clinical Medicine University of Florence Italy
Ion channels are emerging to be particularly relevant in tumours, where their activity can regulate different cancer hallmarks (e.g., cell proliferation and survival, cell invasiveness, and pro-angiogenic programs), hence contributing to drive tumour progression progression. Ion channels are involved in tumor progression through different mechanisms. For example, $\mathrm{K}+$ channels allow uncontrolled tumor cell proliferation by setting the membrane potential $(\mathrm{Vm})$ to rather depolarized values. The $\mathrm{Ca} 2+$-dependent $\mathrm{K}+$ channels can couple $\mathrm{Vm}$ to variations of the intracellular $\mathrm{Ca} 2+$ concentration $([\mathrm{Ca} 2+] \mathrm{i})$. Voltagegated sodium $(\mathrm{NaV})$ channels, besides contributing to tumor "electrical excitability", can regulate intracellular $\mathrm{Na}+$ concentrations $[\mathrm{Na}] \mathrm{i}$ and in turn activate $\mathrm{Na}+$-driven exchangers. In any case, $\mathrm{NaV}$ are mainly involved in the triggering of cell invasiveness and metastatic spread. Some ion channels can operate in cancer in a non-canonical way, forming macromolecular complexes on the plasma membrane, with either growth factor or adhesion receptors. Examples are the Transient Receptor Potential (TRP) channels and some voltage dependent $\mathrm{K}+$ channels (KV), such as KV 11.1 (hERG1). Finally, recent evidence has been provided that some ion channels can form complexes with other ion channels or transporters on the plasma membrane of cancer cells. Such cancer-specific multichannel complexes can represent a new paradigm in cancer research, paving the way for the development of novel therapeutic strategies.

Introduction: Neuropathic cancer pain is induced by the damage of nerve. The nerve damage is induced by the treatments of cancer such as chemotherapy, radiotherapy, and surgery. The incidence of neuropathic pain in the patients with cancer is high $(40 \%)$ after the treatments. The excessive $\mathrm{Ca} 2+$ influx has been implicated in several pain states in cancer treatment with chemotherapeutic drugs, including cisplatin. The TRPM2 channel is activated by ADP-ribose oxidative stress and (ADPR) via the activation of poly (ADPR) polymerase (PARP-1) pathways. However, TRPV1 channel is activated in the dorsal root ganglion (DRG) by several stimuli such as hot chili pepper component (capsaicin) and reactive oxygen species (ROS) (Carrasco et al. 2018). The high expression levels of TRPV1 and TRPM2 were reported in the DRG (Yüksel et al. 2017; Leo et al. 2020). The generation of chemotherapeutic druginduced ROS acted DRG death and peripheral pain in rats (Yüksel et al. 2017).

Results: The low selenium level and glutathione peroxidase activity were reported in the patients with cancer. In a recent study, we observed modulator action of selenium on apoptosis and oxidative stress via the modulation of TRPM2 and TRPV1 channels in DRG of fibromyalgiainduced rats (Yüksel et al. 2017). 
The similar protective effects of selenium in the neuropathic pain-induced rats and mice via the treatment of cisplatin, although the neuropathic pain was not observed in the TRPM2 knock out mice in the TRPM2 knockout mice (Unpublished data). In the speak, I will summarize the recent experimental research findings on how the cisplatininduced oxidative stress, TRPM2 and TRPV1 activations are regulated in peripheral pain by the treatment of selenium.

Conclusions: The results indicate that the activations of TRPM2 and TRPV1 have main roles on cisplatin-mediated neuropathic pain, oxidative stress, and apoptosis.

Keywords: Cisplatin; Oxidative stress; Neuropathic pain; Selenium; TRPM2 channel; TRPV1 channel.

\section{IS-29}

\section{CARBONIC ANHYDRASE INHIBITORS: THERAGNOSTIC AGENTS FOR HYPOXIC TUMORS}

\section{Claudiu Supuran}

University of Florence, Dipartimento di Neuroscienze, Psicologia, Area del Farmaco e Salute del Bambino

Carbonic anhydrase (CA, EC 4.2.1.1) isoforms IX and XII are overexpressed in many hypoxic tumors as a consequence of the hypoxia inducible factor (HIF) activation cascade, being present in limited amounts in normal tissues. These enzymes together with many others are involved in the $\mathrm{pH}$ regulation and metabolism of hypoxic cancer cells, and were validated as antitumor targets recently. A multitude of targeting strategies against these enzymes have been proposed. The small molecule inhibitors, small molecule drug conjugates (SMDCs), antibody-drug conjugates (ADACs) or cytokine-drug conjugates and several monoclonal antibodies against CA IX/XII will be discussed. Relevant synthetic chemistry efforts, coupled with a multitude of preclinical studies, demonstrated that CA IX/XII inhibition leads to the inhibition of growth of primary tumors and metastases and depletes cancer stem cell populations, all factors highly relevant in clinical settings. One small molecule inhibitor, sulfonamide SLC0111, is the most advanced candidate, having completed Phase I and being now in Phase Ib/II clinical trials for the treatment of advanced hypoxic solid tumors.

\section{IS-30 \\ OVERCOMING DRUG RESISTANCE: IDENTIFICATION OF MOLECULAR ACTION MECHANISMS OF NEW GENERATION THERAPEUTICS IN CANCER THERAPY}

Burçin Özbekle, Elif Damla Arisan

Gebze Technical University, Inst. of Biotechnology, Gebze, Kocaeli
During the last two decades the remarkable progress has been achieved in cancer therapy, but primary or acquired drug resistance is the still main obstacle in successful treatments. Although different mechanisms considered that are responsible for tumor drug resistance, dysregulation of cellular energetic mechanisms are critical. AMP-activated protein kinase (AMPK) is a well-known major cellular energy sensor, which negatively regulates metabolic pathways. Cyclin-dependent kinase inhibitors (CDK) could lead to activation of AMPK, which led to differential responses in the cells according to their genetic differences. It was shown that CDK4 was a key coordinator between cellular metabolism and cell cycle progression. In our studies we determined that palbociclib and abemaciclib, CDK4/6 inhibitors, may lead to different responses on AMPK activation status. Especially lipid profile of the cells due to alterations on metabolic pathways were dysregulated according to AMPK activation status of pancreatic ductal adenocarcinoma (PDAC) cells following CDK4/6 inhibitor treatment. We have confirmed that AMPK silencing altered drug-mediated lipid biosynthesis pathways in MIA PaCa-2 cells. These alterations were not significant in PANC-1 PDAC cells. These preliminary findings showed that AMPK plays a critical role in CDK4/6 mediated cellular responses in pancreatic cancer cells through lipid biosynthesis pathways. Further research could enlighten the in vivo responses through monitoring lipid profile and fatty acid synthesis pathways, which are significantly altered in patients to evaluate therapy responses. Acknowledgement: This project supported by TUBITAK 1001 program (Project number 118z100).

\section{IS-31}

\section{ANTIBODY-BASED APPROACHES FOR CANCER TREATMENT AND DIAGNOSIS}

Sibel Kalyoncu Uzunlar

Izmir Biomedicine and Genome Center

Antibodies currently represent more than half of all biopharmaceutical products and their use in cancer has been growing steadily. Because nature uses antibodies to fight against diseases, they have many advantages for therapeutic and diagnostic uses such as being highly targetspecific, low immunogenicity, longer half-life and faster clinical approval. Vernier zone residues locate in framework regions of antibodies affecting conformations of CDR loops and they are underrepresented in the literature. In this talk, an antibody engineering approach based on vernier zone has been applied to improve an anti-VEGF antibody fragment. According to our experimental and computational results, stability and surprisingly affinity increased with rationally designed mutation(s) on vernier zone residues. My talk will describe a novel antibody engineering method to improve certain biophysical/biochemical characteristics of antibodies.

Keywords: Antibody, Antibody Engineering, Cancer 


\section{IS-32}

\section{ANOVEREWOFPEDIATRICHEMATOONCOLOGY FROM TODAY TO THE FUTURE WITH CASES}

\author{
Murat Elli \\ İstanbul Medipol University, Child Health and Diseases \\ Department, Turkey
}

The developments in the field of oncology in the last 20 years in the world are surprising. Overall survival of up to $85 \%$ has been achieved in childhood cancers. Our country is very close to this level. Every year, around 3-400,000 children and adolescent cancers are seen in the world. Although $80 \%$ of children with cancer can be cured in developed countries, only $50 \%$ of children with cancer in the world can survive. However, a significant portion of childhood cancers can be treated with conventional drugs at a low cost. Survival exceeding $85 \%$ in childhood cancers is not considered an adequate success. Because every year 2000 children-adolescents die in the USA. In some tumors (diffuse intrinsic brainstem glioma (DIPG), high-grade gliomas, and metastatic sarcomas) have not been sufficiently successful. While the stable disease is successful in the adult, the aim is to cure in the child.

Beyond the number of children who die each year, an important problem is the long-term morbidity burden that reduces quality of life in some childhood cancers.

As a new guide in treatment, "genomic changes" is one of the main researches to determine more effective treatment strategies. The aim is to detect the genomic changes that form the blueprint to "navigate" the proliferation and survival signaling pathways of childhood cancers. Studies on these genomic changes, genes that initiate the oncogenic event or regulate the tumor suppressor role have gained wide use with imatinib. BCR-ABL is used from leukemias (chronic myeloid leukemia and Ph+ ALL -Post HSCT) to Cr GVHD, aggressive desmoid tumor, neuroglial tumors, high garde glial tumors. Thanks to the data obtained with the 'Human Genome Project', which was started in 1990 and completed in 2003, and the rapid developments in technology, Whole Genome Sequencing has now become a test that gives results in a shorter time and is also affordable. Whole Genome Sequencing can be used in patients with genetic heterogeneity or atypical clinical findings, as well as enable the identification of rare/common inherited diseases in an individual, the detection of genetic changes particularly related to cancer, and the detailed determination of personalized treatments. The WES test is ideal for detecting new mutations and identifying atypical manifestations of the disease. In our country, approximately $85 \%$ of the mutations that are caused by consanguineous marriages and known as Mendelian diseases are in these coded exons. Target agents for leukemias, neuroglial tumors and sarcomas open new horizons in therapy.

\section{IS-33 \\ PREDICTIVE AND PROGNOSTIC FACTORS IN THE ERA OF IMMUNOONCOLOGY}

Ahmet Bilici

Istanbul Medipol University, Medical Oncology

Department, İstanbul

Immunotherapies are one of the most important developments in cancer treatment in recent years. Understanding that in many tumors, the immune system is deficient in recognizing and destroying cancer cells, especially T-lymphocytes, has led to the search for drugs to correct this disorder. Thus, the effectiveness of immunotherapies called immune checkpoint inhibitors, especially in the treatment of advanced cancer, has been investigated and it has been shown that they are effective in cancer types such as lung cancer, melanoma and kidney cancer.

On the other hand, despite the potential benefit of modern immunotherapy in all cancer patients, resistance may develop after a certain response has been achieved or in some patients at the very beginning of treatment. Resistance mechanisms in immunotherapies are complex and heterogeneous. Therefore, individualization of potential patients who will benefit from immunotherapies with certain predictive factors at the beginning will increase the success of the treatment. In all immunotherapy studies, tissue-based retrospective biomarker studies have begun to determine treatment strategies today. Thus, both by knowing the prognosis of the patient in advance thanks to some prognostic factors and by using predictive biomarkers, separating the patients into similar resistance mechanisms will guide the use of various immunotherapy combination treatments. As a result, in the immunotherapy period, it is aimed to increase efficacy and reduce toxicity in the future with a biomarker-based adaptive immunotherapy study design.

\section{IS-34 \\ CHANGING PARADIGMA IN CLINICAL ONCOLOGY: WHAT ARE WE EXPECTING FROM THE FUTURE?}

Ömer Fatih Ölmez

İstanbul Medipol University, School of Medicine, Department of Medical Oncology, Turkey

Cancer is one of the main reason of dies in all over the World and focused on to research new therapeutical methods in order to minimize side effects of traditional therapies in last ten years. During cancer progression, tumors become highly heterogeneous, creating a mixed population of cells characterized by different molecular features and different responses to treatments. 
This heterogeneity can be appreciated at both spatial and temporal levels and is a key factor responsible for the development of resistant phenotypes, supported by a selective pressure on treatment administration. Generally, cancer is treated as a spherical and homogeneous disease, and tumors are considered as a whole cell population. Therefore, a deep understanding of these complex phenomena is essential for designing precise and efficient treatments. In recent years, cancer medicine research has made remarkable strides towards more effective, precise, and less invasive cancer treatments. Nanomedicine, coupled with targeted therapy, helps to improve the biodistribution of new or previously tested chemotherapeutic agents around the specific tissue to be treated, while other strategies such as gene therapy, delivery of siRNAs, immunotherapy and antioxidant molecules offer new possibilities to cancer patients. On the other hand, thermal ablation and magnetic hyperthermia are promising alternatives for tumor resection. Finally, radiomic and pathomic approaches assist in the management of large datasets from cancer patients to improve prognosis and outcome. Recently, the most frequent entries on cancer treatments in the clinical trials database (www.clinicaltrials.gov) include the terms targeted therapy, immunotherapy, and gene therapy, particularly highlighting that these are the most popular methodologies under investigation. As our knowledge of cancer-related culinary studies increases, diagnosis and treatment modalities also diversify and become more effective simultaneously with knowledge. Science and progress continue...

\section{IS-35}

\section{NEW ERA FOR CANCER THERAPIES: BIOENGINEERED STEM CELLS AS DELIVERY VEHICLES}

\section{Nihal Karakas}

Department of Medical Biology, School of Medicine, Istanbul Medipol University, Istanbul, Turkey

Cancer Research Center, Research Institute for Health Sciences and Technologies (SABITA), İstanbul Medipol University, İstanbul, Turkey

The standard care of cancer for solid tumor involves surgical resection and adjuvant chemo- and radiotherapy. These traditional treatments often result in several side effects and high tumor recurrence rates. Alternative therapeutic approaches have been developed and one of the effective way is the targeting therapies against cancers. This strategy can provide selective inducing of cancer cells to death, angiogenesis, recrucuitment of immune cells and/ or blocking tumor cell metastasis. One of the promosing cytotoxic fusion proteins is targeted toxins which can be constructed by replacing the receptor binding domain with a cancer selective ligand, an antibody or an antibody fragment. Beside encouraging pre-clinical outcomes, Phase III clinal trials with targeted toxins were failed due to off target delivery, systemic toxicity and half life of the molecules. Afterwards we and others showed that cell based delivery systems provide an option to secrete therapeutics continously and can be directed towards tumor foci specifically. In this context, we developed Pseudomonas exotoxin (PE) fused cytotoxins and examined their efficacy in a panel of cancer cell lines expressing the target receptor. Our results showed that PE cytotoxins can function on any cell line with the cognate receptor. Furthermore, we engineered toxin resistant cells by using CRISPR/Cas9 gene editing tool and showed that these toxin resistant cells can express and secrete functional toxins. Taken together, these results potentiate that cell based delivery of PE cytotoxins that can be harnessed for several cancers.

This work was supported by TÜBİTAK with project number 117S421 (N.K.)7

\section{IS-36 \\ LIVER CANCER STEM CELLS, 2D- AND 3D- CELL CULTURE STUDIES, ORGANOIDS}

\section{Ranan Gulhan Aktas}

Cancer and Stem Cell Research Center, School of Medicine, Maltepe University, Istanbul, Turkey

Liver cancer is the 5 th most common cancer type. It has also been reported as the third most common cause of cancerrelated death. The progression of hepatocellular carcinoma is thought to be driven by cancer stem cells. Thus, there are tremendous efforts to clarify liver cancer stem cells' features, identify them within other tumor cells, and finally develop new therapeutic models that target those cells.

Our studies at Cancer and Stem Cell Research Center in Maltepe University focus on 2D- and 3D- cell culture studies with HepG2 cells, a hepatocellular carcinoma cell line. Our main goal is to understand the effects of microenvironmental changes on the expression of cancer stem cell markers. First, we create various microenvironmental conditions and then investigate the behavior of liver cancer stem cells by using RT-PCR, ELISA, flow cytometry, confocal microscopy, live-cell imaging methodologies, and immunohistochemistry. We mainly focus on the expression of 7 different stem cell markers: CD133, CD24, CD44, Ov6, Oct4, SSEA 4, TRA1. Consequently, we apply principal component analysis and hierarchical clustering for multi-dimensional data visualization. The results demonstrate correlations between genes, pathways, liver cancer stem cells, and carcinogenic markers; presents promising results for the production of new biomarkers and provides early step information for a personalized medicine application in liver cancer treatment.

Liver organoids, 3D organ-like collections of liver cells, create innovative approaches to disease modeling and drug development. They recapitulate the genomic, transcriptomic features of the original tissue. 
$3^{\text {rd }}$ International Cancer and Ion Channels Congress 2021

Biliary organoids include progenitor cells that can differentiate into different type cells in endodermal lineage; including biliary cells, hepatocytes, pancreatic and intestinal cells. At Boston Children's Hospital Harvard Medical School, we have performed another project aiming long-term establishment of organoids from liver transplant patients. Our results demonstrate a direct effect of dexamethasone on the nuclear expression of Sox9, which is a critical stem cell marker in biliary system.

In conclusion, both $2 \mathrm{D}$ - and $3 \mathrm{D}$ - cell culture techniques serve as great tools to investigate cancer stem cells. In addition, organoids have brought new capabilities and better performance to the laboratories since they mimic the "in-vivo" world and hold remarkable promise for disease modeling and personalized medicine. Finally, cancer organoids may improve our understanding of cancer stem cell resistance and can be used to develop novel cancer treatments.

Keywords: Liver, Cancer, Stem Cells, Organoids, HepG2 cells

IS-37

THE ROLE OF MEDICINAL PLANTS AND HONEYBEE PRODUCTS IN PREVENTION AND TREATMENT OF CANCER

Abdurrahim Kocyigit

Medical Biochemistry, Medical Faculty, Bezmialem Vakif University

Cancer, which is the second cause of death after cardiovascular diseases, is an important health problem all over the world. Oxidative stress and mutations caused by reactive oxygen species (ROS) play an important role in the pathogenesis of cancer. Since conventional methods such as chemotherapy and radiotherapy are often insufficient in cancer treatment, studies for alternative treatment methods are continuing. In recent years, studies on phototherapy and apitherapy products used in traditional medicine have increased in relation to the prevention and treatment of cancer. The therapeutic properties of these products are related to their phenolic content. Phenolic compounds have antioxidant properties. However, in the presence of transition metals such as free iron and copper, they act as pro-oxidant in a dose dependent manner. They also have anti-inflammatory and immunomodulatory effects. Therefore, antioxidant / pro-oxidant, anti-inflammatory, and immune-modulatory effects of these products may be effective in both prevention and treatment of cancer. Although, high doses of phenolic compounds are needed for the pro-oxidant and cancer therapeutic effects, their bioavailability is quite low. Therefore, these substances are need to be administered parenterally or loaded into nano-carriers in order to be used in cancer therapy. In this presentation, the products commonly used in phototherapy and apitherapy for the prevention and treatment of cancer will be discussed in the light of our scientific studies and literature.

Keywords: Antioxidant, apitherapy, cancer, phenolic compounds, phytotherapy, pro-oxidant

\section{IS-38 \\ INTEGRATIVE ONCOLOGY IN PROSTATE CANCER}

Ömer Küçük

Professor of Hematology and Medical Oncology

Winship Cancer Institute of Emory University, Atlanta, Georgia, USA

Integrative oncology is a patient-centered, evidenceinformed field of cancer care that utilizes mind and body practices, natural products, and/or lifestyle modifications from different traditions alongside conventional cancer treatments. Integrative oncology aims to optimize health, quality of life, and clinical outcomes across the cancer care continuum and to empower people to prevent cancer and become active participants before, during, and beyond cancer treatment.

Natural products and botanicals may be useful as complementary therapies for the prevention of adverse effects of radiation and chemotherapy including prevention of second primary tumors, cognitive decline, cardiac toxicity, myelosuppression, pulmonary toxicity, neurotoxicity, nephrotoxicity, and hepatotoxicity. They also may increase the efficacy of chemotherapy and radiation therapy and targeted therapy. Natural and botanical products are generally safe and orally bioavailable.

Epidemiologic studies show an inverse association between dietary lycopene and soy intake and prostate cancer risk. Genistein and daidzein are the most abundant isoflavones in soy. Genistein has activity against a variety of cancer cells in culture, animal model and clinical studies. Genistein's mechanisms of action include antioxidant effects preventing DNA damage, anti-inflammatory effects (IL-1, IL-6 inhibition), epigenetic effects through DNA demethylation and histone acetylation, inhibition of NFkB, RANKL, VEGF, MMP, and EMT.

Healthy lifestyle, physical activity, stress reduction and botanicals may benefit prostate cancer patients before, during and after therapy.

\section{IS-39}

FROM MECHANISMS OF DRUG RESISTANCE TO DRUG DISCOVERY IN THE ERA OF PRECISION MEDICINE

\section{Ozgur Sahin}

Department of Drug Discovery and Biomedical Sciences, University of South Carolina, Columbia, SC, 29208, USA 
$3^{\text {rd }}$ International Cancer and Ion Channels Congress 2021

Our laboratory focuses on elucidating the molecular mechanisms of cancer therapeutics, drug resistance and metastasis using systems biology approaches. We combine our expertise in functional transcriptomics/ proteomics, cancer cell biology and drug discovery with those of the experts from medicinal chemistry, pathology, and bioinformatics to develop novel cancer therapeutics against targets conferring drug resistance in aggressive cancers. In this seminar, I will first talk about overcoming chemotherapy resistance in triple negative breast cancer (TNBC), the most aggressive breast cancer subtype. We identified hypoxia-induced ECM re-modeler, lysyl oxidase (LOX) as a key inducer of chemoresistance, and we showed that inhibiting LOX overcomes chemoresistance. Currently available LOX inhibitors suffer from lack of specificity and high toxicity. To identify more potent and selective LOX inhibitors, we performed a high-throughput screen (HTS) of a diversified small-molecule library and performed structure-activity relationship (SAR) analyses and optimized our lead compound for more potent activity and better drug-like properties. In the second part of the seminar, I will talk about identification and characterization of our potent transforming acidic coiled-coil 3 (TACC3) inhibitor in highly aggressive cancers. Here, we identified a new TACC3-targeting chemotype, BO-264 which demonstrated remarkable antiproliferative activity via spindle assembly checkpoint-dependent mitotic arrest, DNA damage, and apoptosis in the NCI-60 cell line panel. Importantly, BO-264 significantly impaired tumor growth in highly aggressive, treatment-refractory breast and colon cancer mouse models without any major toxicity. We are now developing this chemotype to a more drug-like molecule through ADME, PK and other detailed studies.

\section{IS-40 \\ DIFFERENTIAL EXPRESSION OF MEIS1/2/3 CORRELATES WITH PROSTATE CANCER VIABILITY IN RESPONSE TO MEISI TREATMENT}

$\underline{\text { Fatih Kocabas }}^{1}$, Birkan Girgin ${ }^{1}$, Dilek Telci ${ }^{1}$, Yurdanur Sullu $^{2}$, Kaan Ozturk ${ }^{3}$

${ }^{1}$ Department of Genetics and Bioengineering, Faculty of Engineering, Yeditepe University, Istanbul, Turkey

${ }^{2}$ Surgical Medical Sciences Department, Faculty of Medicine, Ondokuz Mayis University, Samsun, Turkey ${ }^{3}$ Institute of Cellular Virology, ZMBE, University of Munster, Munster, Germany

Prostate cancer (PCa) is the second most diagnosed cancer in males. Understanding the molecular mechanism and investigation of novel ways to block PCa growth or metastasis are vital and a medical necessity. In this study, we examined differential expression of MEIS1/2/3 and its associated factors in $\mathrm{PCa}$ cell lines. MEIS1/2/3 content, reactive oxygen species, cell cycle status, metastatic activities were analyzed in PCa cells pre and post MEIS inhibitor (MEISi) treatments, which is developed in our laboratory as a first-in-class small molecule inhibitor. A correlation was detected between MEIS content and MEISi IC50 values of PCa cells. MEISi decreased the viability of PC-3, DU145, 22Rv-1 and LNCaP cells, and significantly increased apoptosis in parallel with the increased cellular ROS content. The efficacy of MEISi was shown to positively correlate with the levels of MEIS1/2/3 proteins and the long term exposure to MEISi elevated MEIS1/2/3 protein content in $\mathrm{PCa}$ cells. Intriguingly, administration of MEISi to PC-3 and 22Rv1 xenograft models in SCID mice showed that MEISi was not sufficient to block in vivo tumor growth and metastasis. Our findings suggest that MEISi could be used to target PCa with high MEIS expression to lower PCa viability and growth, however, further studies are needed to achieve in vivo efficacy.

Keywords: Prostate cancer, MEIS, MEIS inhibitor, MEISi-2

\section{IS-41 \\ INTERACTOME ANALYSIS OF PROTEIN- PROTEIN INTERACTION NETWORKS REVEALS CRITICAL SIGNALING HUBS FOR THERAPEUTIC TARGETING IN CANCER CELLS}

\section{Gizem Dinler Doğanay}

Istanbul Technical University, Department of Molecular Biology and Genetics, İstanbul

In all cellular events occurring in the cell, there are key tasks performed by dynamic protein-protein interactions (PPI), which provide functional flexibility and stimulate cellular responses to environmental conditions. Loss of function in even one of the PPI members could lead significant systemic alterations that disrupt the linked cellular networks and cause the disease phenotype. By mapping these interaction networks, which play key roles in the cell, it will be possible to understand the disease formation mechanisms leading to the determination of targets for treatment in future. In this respect, experimental determination of PPIs under different cellular conditions, and studying the interaction surfaces at the atomic level according to the cellular need, is crucial for discovery of small molecules, peptides and large molecules that could disrupt these interactions.

In this line, we used a bait protein, called Bag-1, to fish out various interaction networks in critical cellular mechanisms. Bag-1 is a multifunctional protein and frequently overexpressed in breast carcinoma. Bag-1 involves in a wide variety of cellular processes through direct interaction with Hsp70, Raf-1 kinase, the anti-apoptotic Bcl-2, nuclear hormone receptors and ubiquitylation/proteasome machinery components. Three major isoforms of Bag-1, each with a distinct $\mathrm{N}$-termini, include Bag-1S (33 kDa), Bag-1M (46 kDa) and Bag-1L (52 kDa). All Bag-1 isoforms exist with an integrated ubiquitin-like domain 
(ULD) to contact with proteasome components for degradation of specific target proteins and a $\mathrm{COOH}-$ terminal evolutionarily conserved BAG domain to interact and modulate the activity of Hsp70. We, in our previous studies, showed that Bag-1 has a potential role in various protein quality control machines by acting together with a $\mathrm{AAA}+\mathrm{ATPase}, \mathrm{VCP} / \mathrm{p} 97$. Here, we explored the interaction details of Bag-1S with its partners using a combination of various conventional molecular biology techniques and further investigated the interaction surface of Bag-1 with $\mathrm{VCP} / \mathrm{p} 97$ by hydrogen deuterium exchange mass-spectrometry (HDX-MS). We believe targeting the interaction surfaces of adaptor proteins like Bag-1 with its target partners in cancer signaling pathways will offer potential druggable sites for therapeutic intervention.

This work is supported by a grant from TUBITAK, $117 Z 848$.

\section{IS-42}

\section{SPHINGOLIPID METABOLISM AND SIGNALING IN CANCER THERAPY}

\section{Besim Öğretmen}

South Carolina University, Department of Biochemistry \& Molecular Biology

Sphingolipids, including the two central bioactive lipids ceramide and sphingosine 1-phosphate (S1P), have opposing roles regulating cancer cell death and survival, respectively, and there have been exciting developments in understanding how sphingolipid metabolism and signaling regulate these processes in response to anti-cancer therapy, including immunotherapy. Recent studies have provided mechanistic details of the roles of sphingolipids and their downstream targets in the regulation of tumor growth and response to chemotherapy, radiotherapy and/or immunotherapy using innovative molecular, genetic and pharmacological tools to target sphingolipid signaling nodes in cancer cells. For example, structure-function-based studies have provided innovative opportunities to develop mechanism-based anticancer therapeutic strategies to restore anti-proliferative ceramide signaling and/or inhibit pro-survival S1P-S1P receptor (S1PR) signaling. This seminar will summarize how ceramide-induced cellular stress, including aging, mediates cancer cell death through various mechanisms involving the induction of apoptosis, necroptosis and/or mitophagy. Moreover, the metabolism of ceramide for S1P biosynthesis, which is mediated by sphingosine kinase 1 (SPHK1) and SPHK2, and its role in influencing cancer cell growth, drug resistance and tumor metastasis through S1PR-dependent or receptor-independent signaling will be highlighted. Moreover, mechanistic details of agingmediated changes in mitochondrial bioenergetics and lipid metabolism that affect $\mathrm{T}$ cell function will be discussed. For example, ceramide, induced by aging stress, mediates mitophagy, and cell death; however, the aging-related roles of ceramide metabolism in regulating $\mathrm{T}$ cell function remain unknown. Here, we will discuss that activated $\mathrm{T}$ cells isolated from aging mice have elevated C14-/C16ceramide accumulation in mitochondria, generated by ceramide synthase 6 , leading to mitophagy/mitochondrial dysfunction.

Mechanistically, aging-dependent mitochondrial ceramide inhibited protein kinase A, leading to mitophagy in activated $\mathrm{T}$ cells. This aging/ceramide-dependent mitophagy attenuated the anti-tumor functions of $\mathrm{T}$ cells in vitro and in vivo. Also, inhibition of ceramide metabolism or PKA activation by genetic and pharmacologic means prevented mitophagy and restored the central memory phenotype in aging T cells. Thus, these studies help explain the mechanisms behind aging-related dysregulation of $\mathrm{T}$ cells' anti-tumor activity that can be restored by inhibiting ceramide-dependent mitophagy. In addition, biological implications of alterations in ceramide-mediated mitophagy in neurodegenerative disorders will also be summarized.

\section{IS-43 \\ SYSTEMATIC APPROACHES TO STUDY CANCER CELL METABOLISM}

Kıvanç Birsoy

Rockefeller University, Departments of Molecular Biology and Genetics

Metabolic plasticity enables organisms to respond and adapt to changes in their environment. While the core components of most pathways of intermediary metabolism have long been described - consisting of $\sim 3000$ metabolic genes organized in pathways interconnected by $1000 \mathrm{~s}$ of shared metabolites $\neg$, it remains poorly understood how the flow of these metabolites is rewired in different metabolic states. This question is particularly relevant in the context of tumors, as cancer cells are frequently starved for nutrients and exposed to toxic waste products due to a combination of increased nutrient consumption and dysfunctional vasculature. Exploring cancer metabolism also provides a tractable system to address a more fundamental question of how metabolic pathways and extracellular cues cooperate to meet the energetic and biosynthetic needs of cells at different metabolic states of metabolic diseases. In the Birsoy lab, we combine a number of cutting-edge techniques - from the development of forward genetics tools (i.e. CRISPR-Cas9 technology) to metabolomics - to elucidate how cellular metabolism contributes to human disorders such as cancer and inborn errors of metabolism. Our long term research goal is to understand how metabolic pathways in mammalian cells are rewired by their nutrient environment and, further, to determine whether these pathways present metabolic liabilities that could be exploited for disease therapy. 


\section{IS-44 \\ CERAMIDE METABOLISM IN TUMOR METASTASIS}

$\underline{\text { Salih Gencer }}^{1},{ }^{2},{ }^{3}$, Besim Ogretmen ${ }^{3}$

${ }^{1}$ Cancer Research Center, Institute for Health Sciences and Technologies (SABITA), Istanbul Medipol University, Istanbul, Turkey

${ }^{2}$ Istanbul Medipol University, International School of Medicine, Department of Medical Biology, Istanbul, Turkey

${ }^{3}$ Medical University of South Carolina, Biochemistry and Molecular Biology, Charleston, 29401 SC, USA

In the past twenty years, specific sphingolipids have become recognized for their participation in membrane functions and signaling events that control a wide array of cellular processes. Ceramide and sphingosine-1-phosphate, two major sphingolipid metabolites, are involved in signaling pathways that regulate cell proliferation, apoptosis, motility, differentiation, and angiogenesis. These two bioactive sphingolipids play diverse biological functions in some of these cellular processes. Ceramide can be considered a tumor-suppressor lipid, while sphingosine1-phosphate can be considered a tumor-promoting lipid. Response to physiological and pathological processes they can be converted to each other by through a few specific enzymatic reactions (involving of ceramide synthases, sphingosine kinases, and others) (1-3). More recent studies have introduced bioactive sphingolipids and their metabolic enzymes take a role as regulated component in migration and cell mobility which a part of these processes. However, the molecular mechanism of sphingolipids involved is unknown $(4,5)$. Our efforts in our studies, which are serving mechanistically the understanding of bioactive lipids, will be shade some light on this unknown.

In this meeting, I will talk about the therapeutics approaches to metastasis by using novel pathways related to bioactive sphingolipid. Particularly, I will discuss the effect of ceramide syntheses on migration and its related signal pathways in situ and in vivo model with our recent studies. At first part of my talk, briefly, mechanisms that regulate membrane localization of TGF- $\beta$ receptor type I and II (TßRI/II) for the regulation of cell migration/ invasion and metastasis are largely unknown. We identified that alterations in ceramide metabolism by molecular knockdown and/or genetic deletion of ceramide synthase 4, CerS4, reducing bioactive C18-C20-ceramides, results in enhanced TßRI-II signaling at the plasma membrane by inhibiting Smad7 recruitment to the receptor, leading to increased cell migration/invasion via, at least in part, activation of the sonic hedgehog (Shh) pathway. Mechanistically, inhibition of Smad7-TßRI/II interaction in response to CerS4 knockdown led to the activation of the Shh signaling via enhanced association/crosstalk between TßRI-II and smoothened (Smo), a signaling Shh receptor, at the plasma membrane. Also, we found that ceramide synthase 4 (CerS4)-generated ceramide stabilized the association between T $\beta R I$ and the inhibitory factor Smad7, which limited the trafficking of T $\beta R I / I I$ to primary cilia. In reciprocal studies, reconstitution of CerS4/ceramide inhibited TGF-ß-Shh crosstalk, reducing cancer cell migration and inhibition of the cancer cell colonization/ lung metastasis in SCID mice. Moreover, knockdown of CerS4 in cancer cells, but not systemic deletion of CerS4 in mice, increased metastasis of breast cancer cells from mammary pads to the liver, which was inhibited by Smad7 expression or Smo knockdown. Thus, these data reveal that CerS4/ceramide is a novel factor that regulates the recruitment of Smad7 to the TßRI, controlling the stability/ surface expression of the receptor, which modulates TßRI/ II-Smo crosstalk at the plasma membrane of the primary cilium, controlling cell migration and cancer metastasis.

In addition to these data, we also report that a novel mechanism whereby internalization of PDL-1 in response to alterations of lipid/ceramide metabolism by ceramide synthase 4 (CerS4) induces sonic-hedgehog (Shh) signaling to enhance tumor metastasis in triple-negative breast cancers (TNBC), which are highly resistant to immunotherapy. Mechanistically, we show here that internalized PDL-1 in response to CerS4/Ceramide downregulation binds stressgranule-associated Caprin-1, and the PDL-1-Caprin- 1 complex activates Smo and TGF-beta receptor crosstalk/ signaling upon Shh activation to enhance TNBC migration in various TNBC cells. Importantly, the internalization of PDL-1 is mediated by its cytoplasmic domain, including the S278/S279 residues, as their conversion to alanine prevented PDL-1 internalization and Caprin-1 interaction. These data were also validated in enhanced lung metastasis of breast tumors generated in MMTV-PyMT+/+/CerS4/- compared to MMTV-PyMT+/+ mice also consistent with PDL-1-Caprin-1 association and $\mathrm{Shh} / \mathrm{Smo}$ activation in vivo. We also provide data supporting the clinical relevance of the ceramide synthase 4/PDL- 1/Caprin-1/Shh expressions, which negatively affect the survival outcomes of patients with breast cancers.

Keywords: Ceramide, TGF- $\beta$, Primer Cilia, PD-L1, Caprin-1, Tumor Metastasis 